ANNALES

POLONICI MATHEMATICI

$89.2(2006)$

\title{
The Cauchy problem for the coupled Klein-Gordon-Schrödinger system
}

\author{
by Changxing Miao and Youbin Zhu (Beijing)
}

\begin{abstract}
We consider the Cauchy problem for a generalized Klein-Gordon-Schrödinger system with Yukawa coupling. We prove the existence of global weak solutions by the compactness method and, through a special choice of the admissible pairs to match two types of equations, we prove the uniqueness of those solutions by an approach similar to the method presented by J. Ginibre and G. Velo for the pure Klein-Gordon equation or pure Schrödinger equation. Though it is very simple in form, the method has an unnatural restriction on the power of interactions. In the last part of this paper, we use special admissible pairs and Strichartz estimates to remove the restriction, thereby generalizing previous results and obtaining the well-posedness of the system.
\end{abstract}

1. Introduction and the main results. In this paper, we consider the Cauchy problem for a coupled Klein-Gordon-Schrödinger system with Yukawa coupling:

$$
\left\{\begin{array}{l}
i \dot{u}+\Delta u=f_{1}\left(|u|^{2}, v\right) u, \\
\ddot{v}-\Delta v+\mu^{2} v=-\frac{1}{2} f_{2}\left(|u|^{2}, v\right), \\
u(0)=\varphi, \quad v(0)=\psi_{1}, \quad \dot{v}(0)=\psi_{2},
\end{array}\right.
$$

which is a generalization of the system

$$
\left\{\begin{array}{l}
i \dot{u}+\Delta u=-u v, \\
\ddot{v}-\Delta v+\mu^{2} v=|u|^{2}, \\
u(0)=\varphi, \quad v(0)=\psi_{1}, \quad \dot{v}(0)=\psi_{2},
\end{array}\right.
$$

considered in [18] by using a generalized bilinear estimate of Strichartz type and Bourgain's idea [3, 4] to split the data into low and high frequency parts. Systems (1.1) and (1.2) describe a classical model of Yukawa's interaction

2000 Mathematics Subject Classification: 35L05, 35L15.

Key words and phrases: coupled Klein-Gordon-Schrödinger system, Strichartz estimates, global weak solution, uniqueness, well-posedness.

The first author is supported by NSF of China and The Institute of Mathematical Sciences, Chinese University of Hongkong. 
of a conversed complex nucleon field with a neutral real meson field. Here $u$ is a complex scalar nucleon field and $v$ is a real scalar meson field.

A large amount of work has been devoted to the study of Klein-GordonSchrödinger system $[2,8,9,12,14,17,18,22]$, starting from I. Fukuda and M. Tsutsumi [9]. They considered the initial boundary value problem for the K-G-S system under the initial conditions $u(0)=\varphi \in H_{0}^{1}(\Omega) \cap$ $H^{3}(\Omega), v(0)=\psi_{1} \in H_{0}^{1}(\Omega) \cap H^{2}(\Omega), \dot{v}(0)=\psi_{2} \in H_{0}^{1}(\Omega)$ and the boundary conditions $u(x, t)=v(x, t)=0$ for $x \in \partial \Omega$ and $t \in \mathbb{R}$. Here $\Omega$ is a bounded smooth domain in $\mathbb{R}^{3}$. By using Galerkin's method, they proved the existence of global strong solutions under the above conditions. The initial condition on $\varphi$ is unnatural and should be changed into a natural condition such as $\varphi \in H_{0}^{1}(\Omega) \cap H^{2}(\Omega)$.

A second study was done by J.-B. Baillon and J. M. Chadam [2]. They proved the existence of global strong solutions of the initial value problem for the K-G-S system under the initial conditions $\varphi \in H^{2}\left(\mathbb{R}^{3}\right), \psi_{1} \in H^{2}\left(\mathbb{R}^{3}\right)$ and $\psi_{2} \in H^{1}\left(\mathbb{R}^{3}\right)$ by using $L^{p}-L^{q}$ estimates for the elementary solutions of the linear Schrödinger equation and linear Klein-Gordon equation. $L^{p}-L^{q}$ estimates are very useful for the initial value problem for the K-G-S system (see, e.g., A. Bachelot [1]). But they are not available in the case of the initial boundary value problem. Therefore it does not seem that their method is directly applicable to the initial boundary value problems (1.1) and (1.2).

In [14], N. Hayashi and W. Wahl proved the existence of global strong solutions to systems including the K-G-S system under initial conditions as in [2] and boundary conditions as in [9]. They obtained the result by using estimates of the nonlinearity in fractional order Besov spaces developed by P. Brenner and W. Wahl [5], the nonlinear interpolation theorem obtained by W. Wahl [19-21], and the inequality of H. Brezis and T. Gallouët [6] (see also H. Brezis and S. Wainger [7]).

The main purpose of the present paper is to study the well-posedness of the system. We prove the existence of a global weak solution by the compactness method. It is well known that the Schrödinger equation and Klein-Gordon equation have different kinds of admissible pairs; the method used in the proof of uniqueness for a single equation cannot be applied straightforwardly to the coupled case, but through a special choice of the pairs, we prove the uniqueness of solutions by an approach similar to the method presented for the pure nonlinear Schrödinger equation or pure nonlinear Klein-Gordon equation [10,11]. Like the result in [11], we need an unnatural restriction on the power of the interactions. In the last part of this paper, by using special admissible pairs and Strichartz estimates, we remove the restriction on the power, thereby generalizing the result and obtaining the well-posedness of the system. 
First of all, let us recall some of the main notations that will be used in this paper. We denote by $\|\cdot\|_{r}$ the norm in $L^{r}$. Pairs $r, r^{\prime}$ of conjugate indices, where $2 \leq r \leq \infty$, satisfy $1 / r+1 / r^{\prime}=1$. For any integer $k$, we denote by $H^{k} \equiv H^{k}\left(\mathbb{R}^{n}\right)$ the usual Sobolev spaces. For any interval $I \subset \mathbb{R}$, $\bar{I}$ denotes the closure of $I$. For any Banach space $B$, we denote by $\mathcal{C}(I, B)$ the space of strongly continuous functions from $I$ to $B$, by $\mathcal{C}_{w}(I, B)$ the space of weakly continuous functions from $I$ to $B$, and by $\mathcal{C}^{\alpha}(I, B), 0<\alpha<1$, (resp. $\left.\mathcal{C}^{L}(I, B)\right)$ the space of functions from $I$ to $B$ that are strongly Hölder continuous with exponent $\alpha$ (resp. strongly Lipschitz continuous), uniformly on any compact subinterval of $I$. For any $q, 1 \leq q \leq \infty$, we denote by $L^{q}(I, B)$ (resp. $\left.L_{\text {loc }}^{q}(I, B)\right)$ the space of measurable functions $\varphi$ from $I$ to $B$ such that $\|\varphi(\cdot)\|_{B} \in L^{q}(I)$ (resp. $\left.\|\varphi(\cdot)\|_{B} \in L_{\mathrm{loc}}^{q}(I)\right)$.

To obtain the existence of a global weak solution of system (1.1), we shall need the following assumptions on the interactions $f_{1}$ and $f_{2}$ :

(H1) $f_{1}(\chi, \lambda), f_{2}(\chi, \lambda) \in \mathcal{C}\left(\mathbb{R}^{+} \times \mathbb{R}, \mathbb{R}\right)$ and for some $p, 1 \leq p<\infty$, and all $\chi \in \mathbb{R}^{+}, \lambda \in \mathbb{R}$,

$$
\left\{\begin{array}{l}
\left|f_{1}(\chi, \lambda) \chi^{1 / 2}\right| \leq C\left(\chi^{p / 2}+|\lambda|^{p}\right) \\
\left|f_{2}(\chi, \lambda)\right| \leq C\left(\chi^{p / 2}+|\lambda|^{p}\right)
\end{array}\right.
$$

$\left(\mathrm{H} 1^{\prime}\right) f_{1}(\chi, \lambda), f_{2}(\chi, \lambda) \in \mathcal{C}\left(\mathbb{R}^{+} \times \mathbb{R}, \mathbb{R}\right)$ and for some $p, 1 \leq p<\infty$, and all $\chi \in \mathbb{R}^{+}, \lambda \in \mathbb{R}$,

$$
\left\{\begin{array}{l}
\left|f_{1}(\chi, \lambda) \chi^{1 / 2}\right| \leq C\left(\chi^{p / 2}+\chi^{1 / 2}+|\lambda|^{p}+|\lambda|\right) \\
\left|f_{2}(\chi, \lambda)\right| \leq C\left(\chi^{p / 2}+\chi^{1 / 2}+|\lambda|^{p}+|\lambda|\right)
\end{array}\right.
$$

(H2) There exists a function $F(\chi, \lambda) \in \mathcal{C}^{1}\left(\mathbb{R}^{+} \times \mathbb{R}, \mathbb{R}\right)$ such that

$$
\frac{\partial F(\chi, \lambda)}{\partial \chi}=f_{1}(\chi, \lambda), \quad \frac{\partial F(\chi, \lambda)}{\partial \lambda}=f_{2}(\chi, \lambda) ;
$$

what is more, $F(\chi, \lambda)$ satisfies the estimate

$$
F(\chi, \lambda) \geq-\mu_{1}^{2}|\lambda|^{2}-C_{0} \chi^{\left(p_{1}+1\right) / 2}
$$

for some $C_{0}>0, \mu>\mu_{1}$ and $1 \leq p_{1}<1+4 / n$.

In particular, (H1), $\left(\mathrm{H}^{\prime}\right)$ and (H2) hold for $\chi=|u|^{2}$ and $\lambda=v$, and it is easy to see that $\left(\mathrm{H}^{\prime}\right)$ is more general than $(\mathrm{H} 1)$.

As is well known, there is a so-called critical growth condition on $p$ to prove the uniqueness. Even if it holds, the above assumptions are still not enough to obtain the uniqueness of the global weak solutions obtained. So we shall introduce the following stronger assumptions on the interactions $f_{1}$ and $f_{2}$ for $1 \leq p<1+4 /(n-2)$ : 
(H3) $f_{1}\left(|u|^{2}, v\right), f_{2}\left(|u|^{2}, v\right)$ satisfy

$$
\left\{\begin{array}{l}
\left|f_{1}\left(\left|u_{1}\right|^{2}, v_{1}\right) u_{1}-f_{1}\left(\left|u_{2}\right|^{2}, v_{2}\right) u_{2}\right| \\
\quad \leq C\left(\left|u_{1}\right|^{p-1}+\left|u_{2}\right|^{p-1}+\left|v_{1}\right|^{p-1}+\left|v_{2}\right|^{p-1}\right)\left(\left|u_{1}-u_{2}\right|+\left|v_{1}-v_{2}\right|\right), \\
\left|f_{2}\left(\left|u_{1}\right|^{2}, v_{1}\right)-f_{2}\left(\left|u_{2}\right|^{2}, v_{2}\right)\right| \\
\quad \leq C\left(\left|u_{1}\right|^{p-1}+\left|u_{2}\right|^{p-1}+\left|v_{1}\right|^{p-1}+\left|v_{2}\right|^{p-1}\right)\left(\left|u_{1}-u_{2}\right|+\left|v_{1}-v_{2}\right|\right) .
\end{array}\right.
$$

$\left(\mathrm{H} 3^{\prime}\right) f_{1}\left(|u|^{2}, v\right), f_{2}\left(|u|^{2}, v\right)$ satisfy

$$
\left\{\begin{array}{l}
\left|f_{1}\left(\left|u_{1}\right|^{2}, v_{1}\right) u_{1}-f_{1}\left(\left|u_{2}\right|^{2}, v_{2}\right) u_{2}\right| \\
\quad \leq C\left(1+\left|u_{1}\right|^{p-1}+\left|u_{2}\right|^{p-1}+\left|v_{1}\right|^{p-1}+\left|v_{2}\right|^{p-1}\right)\left(\left|u_{1}-u_{2}\right|+\left|v_{1}-v_{2}\right|\right), \\
\quad\left|f_{2}\left(\left|u_{1}\right|^{2}, v_{1}\right)-f_{2}\left(\left|u_{2}\right|^{2}, v_{2}\right)\right| \\
\quad \leq C\left(1+\left|u_{1}\right|^{p-1}+\left|u_{2}\right|^{p-1}+\left|v_{1}\right|^{p-1}+\left|v_{2}\right|^{p-1}\right)\left(\left|u_{1}-u_{2}\right|+\left|v_{1}-v_{2}\right|\right) .
\end{array}\right.
$$

After all these preparations, our main results are the following:

TheOrem 1. Let $f_{1}\left(|u|^{2}, v\right), f_{2}\left(|u|^{2}, v\right)$ satisfy $(\mathrm{H} 1)$ or $\left(\mathrm{H} 1^{\prime}\right)$ and $(\mathrm{H} 2)$. If $p+1>2^{*}$, assume in addition that

$$
F\left(|u|^{2}, v\right) \geq-\mu_{1}^{2}|v|^{2}+C\left(|u|^{p+1}+|v|^{p+1}\right)
$$

for some $C>0$. Let $X=H^{1} \cap L^{p+1}, 2^{*}=2 n /(n-2)$ and

$$
\beta(r)=2 \alpha(r)=1-\delta(r) \min \left(1, \frac{1}{\delta(p+1)}\right), \quad \delta(r)=\frac{n}{2}-\frac{n}{r} .
$$

Let $\left(\varphi, \psi_{1}, \psi_{2}\right) \in X \times X \times L^{2}$. Then (1.1) has a global weak solution $(u, v) \in$ $\mathcal{C}_{w}(\mathbb{R}, X) \times \mathcal{C}_{w}(\mathbb{R}, X)$ such that

$$
\begin{gathered}
u \in L^{\infty}(\mathbb{R}, X) \cap \bigcap_{2 \leq r<\max \left(p+1,2^{*}\right)} \mathcal{C}^{\alpha(r)}\left(\mathbb{R}, L^{r}\right), \\
v \in L_{\mathrm{loc}}^{\infty}(\mathbb{R}, X) \cap \mathcal{C}^{L}\left(\mathbb{R}, L^{2}\right) \cap \bigcap_{2<r<\max \left(p+1,2^{*}\right)} \mathcal{C}^{\beta(r)}\left(\mathbb{R}, L^{r}\right), \\
\dot{v} \in L_{\mathrm{loc}}^{\infty}\left(\mathbb{R}, L^{2}\right) \cap \mathcal{C}_{w}\left(\mathbb{R}, L^{2}\right) \cap \mathcal{C}^{L}\left(\mathbb{R}, X^{\prime}\right) .
\end{gathered}
$$

If $p+1 \geq 2^{*}$, assume in addition that $F$ can be decomposed as $F=F_{1}+F_{2}$ where $F_{1}$ satisfies $(\mathrm{H} 1)$ or $\left(\mathrm{H}^{\prime}\right)$ and $(\mathrm{H} 2)$ for some $\bar{p}, 1 \leq \bar{p}<p$, and $F_{2}$ is weakly lower semicontinuous. Then

$$
\begin{gathered}
\|u(t)\|_{2}=\|\varphi\|_{2} \quad \text { for all } t \in \mathbb{R}, \\
E(u, v, \dot{v}) \leq E\left(\varphi, \psi_{1}, \psi_{2}\right) .
\end{gathered}
$$

TheOREm 2. Let $f_{1}\left(|u|^{2}, v\right), f_{2}\left(|u|^{2}, v\right)$ satisfy (H3), and

$$
1+\frac{2}{n-1} \leq p<1+\frac{4}{n-2}-\frac{2}{(n+1)(n-2)} .
$$


Then there exists at most one pair $(u, v) \in L^{\infty}\left(I, H^{1}\right) \times L^{\infty}\left(I, H^{1}\right)$ which satisfies (1.1) in $\mathcal{D}^{\prime}\left(I, H^{1}\right) \times \mathcal{D}^{\prime}\left(I, H^{1}\right)$.

TheOREM 3. Let $f_{1}\left(|u|^{2}, v\right), f_{2}\left(|u|^{2}, v\right)$ satisfy $\left(\mathrm{H}^{\prime}\right)$. Then there exists at most one pair $(u, v) \in L^{\infty}\left(I, H^{1}\right) \times L^{\infty}\left(I, H^{1}\right)$ which satisfies (1.1) in $\mathcal{D}^{\prime}\left(I, H^{1}\right) \times \mathcal{D}^{\prime}\left(I, H^{1}\right)$.

This paper is organized as follows. In Section 2, we prove the existence of a global weak solution by the compactness method. Under the stronger assumption (H3), by using the same strategy for the two equations of the system, we prove in Section 3 the uniqueness of the solutions obtained in Section 2 for $p$ as in Theorem 2. Though the method is very simple, the restriction on $p$ is unnatural. By choosing two sets of special admissible pairs $(r, q)$, we use a Strichartz estimate to remove the restriction on $p$ under the more general assumption (H3') instead of (H3) in Section 4.

2. Existence of global weak solutions. Now, we are ready to prove the existence of a global weak solution to (1.1) by the compactness method, which can be found in [11]. For the convenience of the reader, we prove it and a related lemma in detail. For $(u, v) \in H^{1} \times H^{1}$ such that $F\left(|u|^{2}, v\right) \in L^{1}$, the energy is defined by

$$
E(u, v, \dot{v})=\|\nabla u\|_{2}^{2}+\|\nabla v\|_{2}^{2}+\|\dot{v}\|_{2}^{2}+\mu^{2}\|v\|_{2}^{2}+\int_{\mathbb{R}^{n}} F\left(|u|^{2}, v\right) d x .
$$

The assumption (H2) formally implies the conservation of the $L^{2}$-norm for $u$ and of the energy.

The dual of $X=H^{1} \cap L^{p+1}$ is denoted by $X^{\prime}=H^{-1}+L^{(p+1) / p}$, the duality being realized through the $L^{2}$ scalar product $(f, g)$, linear in $g$ and anti-linear in $f$.

Lemma 2.1. Let $f_{1}, f_{2}$ satisfy $(\mathrm{H} 1)$ or $\left(\mathrm{H}^{\prime}\right), I$ be a bounded open interval of $\mathbb{R}$ and $(u, v) \in L^{\infty}(I, X) \times L^{\infty}(I, X)$. Then

(1) $f_{1}\left(|u|^{2}, v\right) u, f_{2}\left(|u|^{2}, v\right) \in L^{\infty}\left(I, L^{2}+L^{(p+1) / p}\right)$.

(2) Let in addition $(u, v)$ satisfy $(1.1)$ in $\mathcal{D}^{\prime}\left(I, X^{\prime}\right) \times \mathcal{D}^{\prime}\left(I, X^{\prime}\right)$. Then

(a) We have

$$
\dot{u} \in L^{\infty}\left(I, X^{\prime}\right), \quad u \in \mathcal{C}_{w}(\bar{I}, X) \cap \bigcap_{2 \leq r<\max \left(p+1,2^{*}\right)} \mathcal{C}^{\alpha(r)}\left(\bar{I}, L^{r}\right)
$$

with $\alpha(r)$ defined by (1.5).

(b) $\ddot{v} \in L^{\infty}\left(I, X^{\prime}\right), \dot{v} \in \mathcal{C}^{L}\left(I, X^{\prime}\right)$. If in addition $\dot{v} \in L^{\infty}\left(I, L^{2}\right)$, then

$$
\dot{v} \in \mathcal{C}_{w}\left(I, L^{2}\right), \quad v \in \mathcal{C}_{w}(\bar{I}, X) \cap \mathcal{C}^{L}\left(\bar{I}, L^{2}\right) \cap \bigcap_{2<r<\max \left(p+1,2^{*}\right)} \mathcal{C}^{\beta(r)}\left(I, L^{r}\right)
$$

with $\beta(r)$ defined by (1.5). 
(3) For any $t, s \in \bar{I},(u, v)$ satisfies the integral system

$$
\left\{\begin{array}{l}
u(t)=U(t-s) u(s)-i \int_{s}^{t} U(t-\tau) f_{1}\left(|u|^{2}, v\right) u(\tau) d \tau \\
v(t)=\dot{K}(t-s) v(s)+K(t-s) \dot{v}(s)-\frac{1}{2} \int_{s}^{t} K(t-\tau) f_{2}\left(|u|^{2}, v\right) d \tau
\end{array}\right.
$$

where the integral is a Bochner integral in $H^{-k}, k \geq \max (1, \delta(p+1))$.

(4) We have

$$
\|u\|_{2}=\text { const }, \quad \forall t \in \bar{I} .
$$

Proof. For simplicity, we only consider the general case $\left(\mathrm{H} 1^{\prime}\right)$ in the proofs of this lemma and the following theorem. For (H1), the proofs are similar but a little simpler.

(1) We decompose $u f_{1}$ as

$$
u f_{1}=f_{11}+f_{12} \triangleq\left(u f_{1}-u f_{1} \chi_{|u|,|v| \leq 1}\right)+u f_{1} \chi_{|u|,|v| \leq 1} .
$$

Then

$$
\left\{\begin{array}{l}
\left|f_{11}\left(|u|^{2}, v\right)\right| \leq C\left(|u|^{p}+|v|^{p}\right) \\
\left|f_{12}\left(|u|^{2}, v\right)\right| \leq C(|u|+|v|)
\end{array}\right.
$$

thus

$$
\left\{\begin{array}{l}
\left|f_{11}\left(|u|^{2}, v\right)\right|^{(p+1) / p} \leq C\left(|u|^{p+1}+|v|^{p+1}\right), \\
\left|f_{12}\left(|u|^{2}, v\right)\right|^{2} \leq C\left(|u|^{2}+|v|^{2}\right) .
\end{array}\right.
$$

Similarly, for $f_{2}=f_{21}+f_{22}$ we have

$$
\left\{\begin{array}{l}
\left|f_{21}\left(|u|^{2}, v\right)\right|^{(p+1) / p} \leq C\left(|u|^{p+1}+|v|^{p+1}\right), \\
\left|f_{22}\left(|u|^{2}, v\right)\right|^{2} \leq C\left(|u|^{2}+|v|^{2}\right) .
\end{array}\right.
$$

Now (1) follows from (2.5), (2.6) and standard measurability arguments.

(2) (a) Since $u \in L^{\infty}(I, X)$, it follows from (1) that

$$
-\Delta u+f_{1}\left(|u|^{2}, v\right) u \in L^{\infty}\left(I, H^{-1}+L^{(p+1) / p}\right) \hookrightarrow \mathcal{D}^{\prime}\left(I, X^{\prime}\right) .
$$

Note that for all $\psi \in \mathcal{C}_{\mathrm{c}}^{\infty}\left(I, X^{\prime}\right)$,

$$
\langle\dot{u}, \psi\rangle=-\langle u, \dot{\psi}\rangle \leq \sup _{t \in I}\|u\|_{X}\|\dot{\psi}\|_{X^{\prime}}<\infty,
$$

we have

$$
\dot{u} \in \mathcal{D}^{\prime}(I, X) \hookrightarrow \mathcal{D}^{\prime}\left(I, X^{\prime}\right) .
$$

Since $(u, v)$ satisfies $(1.1)$ in $\mathcal{D}^{\prime}\left(I, X^{\prime}\right) \times \mathcal{D}^{\prime}\left(I, X^{\prime}\right)$, this together with $(2.7)$ and (2.8) implies

$$
\dot{u}=-i\left(-\Delta u+f_{1}\left(|u|^{2}, v\right) u\right) \in L^{\infty}\left(I, X^{\prime}\right) .
$$


From $u \in L^{\infty}(I, X) \hookrightarrow L^{\infty}\left(I, X^{\prime}\right)$ and (2.9) we obtain

$$
\|u(t)-u(s)\|_{X^{\prime}}=\left\|\int_{0}^{1} \dot{u}(\theta t+(1-\theta) s)(t-s) d \theta\right\|_{X^{\prime}} \leq|t-s|\|\dot{u}\|_{L^{\infty}\left(I, X^{\prime}\right)},
$$

so $u \in \mathcal{C}^{L}\left(\bar{I}, X^{\prime}\right) \hookrightarrow \mathcal{C}_{w}\left(\bar{I}, X^{\prime}\right)$. Together with the fact that $u \in L^{\infty}(I, X)$ and Lions' lemma [15], it follows that $u \in \mathcal{C}_{w}(\bar{I}, X)$.

We can approximate $u, v$ by sequences $\left\{u_{j}\right\}$ and $\left\{v_{j}\right\}$ in $\mathcal{C}_{\mathrm{c}}^{\infty}(\mathbb{R}, X)$ such that, after restriction to $I, u_{j}$ converges to $u$ in $L^{2}(I, X)$ and $\dot{u}_{j}$ converges to $\dot{u}$ in $L^{2}\left(I, X^{\prime}\right)$, and similarly for $v$ and $v_{j}$. This implies that $u \in \mathcal{C}\left(\bar{I}, L^{2}\right), v \in$ $\mathcal{C}\left(\bar{I}, L^{2}\right)$, and $u_{j}$ and $v_{j}$ tend to $u$ and $v$ respectively in $\mathcal{C}\left(\bar{I}, L^{2}\right)$. In fact, since $u \in L^{2}(\bar{I}, X) \hookrightarrow L^{2}\left(\bar{I}, X^{\prime}\right)$ and $\dot{u} \in L^{2}\left(\bar{I}, X^{\prime}\right)$, we can easily get $u \in \mathcal{C}\left(\bar{I}, X^{\prime}\right)$, and then

$$
\begin{aligned}
\|u(t)-u(s)\|_{2} & \leq\|u(t)-u(s)\|_{X}^{\theta}\|u(t)-u(s)\|_{X^{\prime}}^{1-\theta} \\
& \leq 2^{\theta}\|u\|_{L^{\infty}(I, X)}^{\theta}\|u(t)-u(s)\|_{X^{\prime}}^{1-\theta} \\
& \rightarrow 0 \quad(t \rightarrow s)
\end{aligned}
$$

i.e., $u \in \mathcal{C}\left(\bar{I}, L^{2}\right)$.

Letting $j \rightarrow \infty$ on both sides of the identity

$$
\left\|u_{j}(t)-u_{j}(s)\right\|_{2}^{2}=2 \int_{s}^{t} \operatorname{Re}\left(\dot{u}_{j}(\tau), u_{j}(\tau)-u_{j}(s)\right) d \tau
$$

we obtain

$$
\|u(t)-u(s)\|_{2}^{2} \leq 4|t-s|\|\dot{u}\|_{L^{\infty}\left(I, X^{\prime}\right)}\|u\|_{L^{\infty}(I, X)} .
$$

Thus $u(t) \in \mathcal{C}^{1 / 2}\left(\bar{I}, L^{2}\right)$. By interpolation, we have

$$
\left\{\begin{array}{l}
\left(\mathcal{C}^{1 / 2}\left(\bar{I}, L^{2}\right), L^{\infty}\left(\bar{I}, H^{1}\right)\right)_{\theta_{1}} \sim \mathcal{C}^{\alpha_{1}(r)}\left(\bar{I}, L^{r}\right) \\
\left(\mathcal{C}^{1 / 2}\left(\bar{I}, L^{2}\right), L^{\infty}\left(\bar{I}, L^{p+1}\right)\right)_{\theta_{2}} \sim \mathcal{C}^{\alpha_{2}(r)}\left(\bar{I}, L^{r}\right)
\end{array}\right.
$$

where

$$
\frac{\theta_{1}}{2}+\frac{1-\theta_{1}}{2^{*}}=\frac{1}{r}, \quad \frac{\theta_{2}}{2}+\frac{1-\theta_{2}}{p+1}=\frac{1}{r}
$$

So

$$
\alpha_{1}=\frac{\theta_{1}}{2}=\frac{1}{2}(1-\delta(r)), \quad \alpha_{2}=\frac{\theta_{2}}{2}=\frac{1}{2}\left(1-\frac{\delta(r)}{\delta(p+1)}\right)
$$

i.e.,

$$
u \in \bigcap_{2 \leq r<\max \left(p+1,2^{*}\right)} \mathcal{C}^{\alpha(r)}\left(\bar{I}, L^{r}\right)
$$

with $\alpha(r)$ defined by (1.5).

(b) In view of the fact that $v \in L^{\infty}(I, X)$, it follows from (1) that $\left(\Delta v-\mu^{2} v\right)-\frac{1}{2} f_{2}\left(|u|^{2}, v\right) \in L^{\infty}\left(I, H^{-1}+L^{(p+1) / p}\right) \hookrightarrow \mathcal{D}^{\prime}\left(I, X^{\prime}\right)$. 
By similar arguments to (a), we also have

$$
\ddot{v} \in L^{\infty}\left(I, X^{\prime}\right), \quad \dot{v} \in \mathcal{C}^{L}\left(I, X^{\prime}\right), \quad \dot{v} \in \mathcal{C}_{w}\left(I, L^{2}\right), \quad v \in \mathcal{C}_{w}(\bar{I}, X) .
$$

On the other hand,

$$
\|v(t)-v(s)\|_{2}=\left\|\int_{0}^{1} \dot{v}(\theta t+(1-\theta) s)(t-s) d \theta\right\|_{2} \leq|t-s|\|\dot{v}\|_{L^{\infty}\left(I, L^{2}\right)}
$$

implies that $v(t) \in \mathcal{C}^{L}\left(\bar{I}, L^{2}\right)$, and we also have by interpolation

$$
v(t) \in \bigcap_{2<r<\max \left(p+1,2^{*}\right)} \mathcal{C}^{\beta(r)}\left(\bar{I}, L^{r}\right)
$$

with $\beta(r)$ defined by (1.5).

(3) We again approximate $u, v$ by regularized sequences $\left\{u_{j}\right\}$ and $\left\{v_{j}\right\}$ as in (2)(a). The functions $u_{j}$ and $v_{j}$ satisfy

$$
\left\{\begin{array}{c}
u_{j}(t)-U(t-s) u_{j}(s)=\int_{s}^{t} U(t-\tau)\left(\dot{u}_{j}(\tau)-i \Delta u_{j}(\tau)\right) d \tau \\
v_{j}(t)-\dot{K}(t-s) v_{j}(s)-K(t-s) \dot{v}_{j}(s) \\
=\int_{s}^{t} K(t-\tau)\left(\left(\mu^{2}-\Delta\right) v_{j}(\tau)+\ddot{v}_{j}(\tau)\right) d \tau
\end{array}\right.
$$

Letting $j \rightarrow \infty$, for fixed $t$ and $s$ we obtain

$$
\left\{\begin{array}{c}
u_{j}(t)-U(t-s) u_{j}(s) \rightarrow u(t)-U(t-s) u(s), \\
v_{j}(t)-\dot{K}(t-s) v_{j}(s)-K(t-s) \dot{v}_{j}(s) \\
\rightarrow v(t)-\dot{K}(t-s) v(s)-K(t-s) \dot{v}(s)
\end{array}\right.
$$

in $L^{2}$, and

$$
\left\{\begin{array}{c}
\int_{s}^{t} U(t-\tau)\left(\dot{u}_{j}(\tau)-i \Delta u_{j}(\tau)\right) d \tau \rightarrow \int_{s}^{t} U(t-\tau)(\dot{u}(\tau)-i \Delta u(\tau)) d \tau \\
\int_{s}^{t} K(t-\tau)\left(\left(\mu^{2}-\Delta\right) v_{j}(\tau)+\ddot{v}_{j}(\tau)\right) d \tau \\
\rightarrow \int_{s}^{t} K(t-\tau)\left(\left(\mu^{2}-\Delta\right) v(\tau)+\ddot{v}(\tau)\right) d \tau
\end{array}\right.
$$

in $H^{-k}$. Then (2.2) follows from (1.1).

(4) Letting $j \rightarrow \infty$ in the identity

$$
\left\|u_{j}(t)\right\|_{2}^{2}-\left\|u_{j}(s)\right\|_{2}^{2}=\int_{s}^{t} 2 \operatorname{Re}\left(\dot{u}_{j}(\tau), u_{j}(\tau)\right) d \tau
$$

and using the fact that $\operatorname{Re}\left(\dot{u}_{j}, u_{j}\right)=0$ as a consequence of (1.1), yields the result. 
Proof of Theorem 1. Without loss of generality, we consider any compact interval $J$ containing 0 .

STEP 1: Finite-dimensional approximation. Let $\left\{w_{j}\right\}, j \in \mathbb{Z}^{+}$, be an orthonormal basis of $X$. For any $m \in \mathbb{Z}^{+}$, we look for an approximate solution of (1.1) of the form

$$
u_{m}(t)=\sum_{1 \leq k \leq m} g_{m k}(t) w_{k}(x), \quad v_{m}(t)=\sum_{1 \leq k \leq m} h_{m k}(t) w_{k}(x)
$$

with

$$
\left\{\begin{array}{l}
\left(w_{j}, i \dot{u}_{m}+\Delta u_{m}-f_{1}\left(\left|u_{m}\right|^{2}, v_{m}\right) u_{m}\right)=0 \\
\left(w_{j}, \ddot{v}_{m}-\Delta v_{m}+\mu^{2} v_{m}+\frac{1}{2} f_{2}\left(\left|u_{m}\right|^{2}, v_{m}\right)\right)=0
\end{array}\right.
$$

and the initial conditions

$$
\left\{\begin{array}{l}
u_{m}(0)=\varphi_{m}(x)=\sum_{1 \leq k \leq m} C_{m k} w_{k}(x), \\
v_{m}(0)=\psi_{1 m}(x)=\sum_{1 \leq k \leq m} D_{1 m k} w_{k}(x), \\
\dot{v}_{m}(0)=\psi_{2 m}(x)=\sum_{1 \leq k \leq m} D_{2 m k} w_{k}(x),
\end{array}\right.
$$

where $C_{m k}, D_{1 m k}, D_{2 m k}$ are chosen in such a way that

$$
\lim _{m \rightarrow \infty}\left\|\varphi_{m}-\varphi\right\|_{X}=\lim _{m \rightarrow \infty}\left\|\psi_{1 m}-\psi_{1}\right\|_{X}=\lim _{m \rightarrow \infty}\left\|\psi_{2 m}-\psi_{2}\right\|_{2}=0 .
$$

By linear independence of $\left\{w_{j}\right\},(2.10)$ can be put in normal form and by Peano's theorem [13], it has a solution in some interval $\left[-T_{m}, T_{m}\right]$ with $T_{m}>0$. In order to prove that $T_{m}=\infty$, we ought to derive some a priori estimates on the solution of (2.10). Multiplying by $\bar{g}_{m j}, \overline{\dot{g}}_{m j}, \overline{\dot{h}}_{m j}$ and summing over $j$ from 1 to $m$, we obtain

$$
\left\{\begin{array}{l}
\left(u_{m}(t), i \dot{u}_{m}+\Delta u_{m}-f_{1}\left(\left|u_{m}\right|^{2}, v_{m}\right) u_{m}\right)=0, \\
\left(\dot{u}_{m}(t), i \dot{u}_{m}+\Delta u_{m}-f_{1}\left(\left|u_{m}\right|^{2}, v_{m}\right) u_{m}\right)=0, \\
\left(\dot{v}_{m}(t), \ddot{v}_{m}-\Delta v_{m}+\mu^{2} v_{m}+\frac{1}{2} f_{2}\left(\left|u_{m}\right|^{2}, v_{m}\right)\right)=0,
\end{array}\right.
$$

therefore

$$
\left\|u_{m}(t)\right\|_{2}^{2}=\left\|\varphi_{m}\right\|_{2}^{2}
$$

and

$$
\begin{aligned}
& \left\|\nabla u_{m}\right\|_{2}^{2}+\left\|\nabla v_{m}\right\|_{2}^{2}+\left\|\dot{v}_{m}\right\|_{2}^{2}+\mu^{2}\left\|v_{m}\right\|_{2}^{2}+\int_{\mathbb{R}^{n}} F\left(\left|u_{m}\right|^{2}, v_{m}\right) d x \\
= & \left\|\nabla \varphi_{m}\right\|_{2}^{2}+\left\|\nabla \psi_{1 m}\right\|_{2}^{2}+\left\|\psi_{2 m}\right\|_{2}^{2}+\mu^{2}\left\|\psi_{1 m}\right\|_{2}^{2}+\int_{\mathbb{R}^{n}} F\left(\left|\varphi_{m}\right|^{2}, \psi_{1 m}\right) d x \\
\leq & \|\nabla \varphi\|_{2}^{2}+\left\|\nabla \psi_{1}\right\|_{2}^{2}+\left\|\psi_{2}\right\|_{2}^{2}+\mu^{2}\left\|\psi_{1}\right\|_{2}^{2}+\int_{\mathbb{R}^{n}} F\left(|\varphi|^{2}, \psi_{1}\right) d x .
\end{aligned}
$$


Since

$$
\sum_{1 \leq k \leq m}\left|g_{m k}\right|^{2} \leq C\left\|u_{m}\right\|_{2}^{2}<\infty, \quad \sum_{1 \leq k \leq m}\left|h_{m k}\right|^{2} \leq C\left\|v_{m}\right\|_{2}^{2}<\infty,
$$

by Picard's theorem, (2.12)-(2.14) imply the existence of a global solution of (2.10), that is, $T_{m}=\infty$.

STEP 2: Uniform estimates on $u_{m}, v_{m}$. In order to take the limit as $m \rightarrow \infty$, we need stronger uniform estimates on $u_{m}$ and $v_{m}$.

CASE 1: $p+1 \leq 2^{*}$. We have proved in Step 1 that

$$
\left\|u_{m}\right\|_{2}^{2}=\left\|\varphi_{m}\right\|_{2}^{2}, \quad E\left(u_{m}, v_{m}, \dot{v}_{m}\right)=E\left(\varphi_{m}, \psi_{1 m}, \psi_{2 m}\right),
$$

hence from $(\mathrm{H} 2)$,

$$
\begin{aligned}
& \left\|\varphi_{m}\right\|_{2}^{2}+E\left(\varphi_{m}, \psi_{1 m}, \psi_{2 m}\right) \\
& =\left\|u_{m}\right\|_{2}^{2}+\left\|\nabla u_{m}\right\|_{2}^{2}+\left\|\nabla v_{m}\right\|_{2}^{2}+\left\|\dot{v}_{m}\right\|_{2}^{2}+\mu^{2}\left\|v_{m}\right\|_{2}^{2}+\int_{\mathbb{R}^{n}} F\left(\left|u_{m}\right|^{2}, v_{m}\right) d x \\
& \geq\left\|u_{m}\right\|_{2}^{2}+\left\|\nabla u_{m}\right\|_{2}^{2}+\left\|\nabla v_{m}\right\|_{2}^{2}+\left\|\dot{v}_{m}\right\|_{2}^{2} \\
& \quad+\left(\mu^{2}-\mu_{1}^{2}\right)\left\|v_{m}\right\|_{2}^{2}-C_{0} \int_{\mathbb{R}^{n}}\left|u_{m}\right|^{p_{1}+1} d x .
\end{aligned}
$$

By direct computation, we have

$$
-C_{0} \int_{\mathbb{R}^{n}}|u|^{p_{1}+1} d x \geq-\frac{1}{2}\|\nabla u\|_{2}^{2}-A\|u\|_{2}^{B},
$$

where

$$
A=C_{0} \frac{2-\left(p_{1}+1\right) \delta\left(p_{1}+1\right)}{2 \varepsilon_{0}^{\frac{2-\left(p_{1}+1\right) \delta\left(p_{1}+1\right)}{2}}}>0, \quad B=\frac{2\left(p_{1}+1\right)\left(1-\delta\left(p_{1}+1\right)\right)}{2-\left(p_{1}+1\right) \delta\left(p_{1}+1\right)},
$$

with $\varepsilon_{0}$ small enough that

$$
\frac{\varepsilon_{0}^{a}}{a} \leq \frac{1}{2 C_{0}} \quad \text { for } \quad a=\frac{2}{\left(p_{1}+1\right) \delta\left(p_{1}+1\right)},
$$

thus

$$
\begin{gathered}
\left\|\varphi_{m}\right\|_{2}^{2}+E\left(\varphi_{m}, \psi_{1 m}, \psi_{2 m}\right) \geq \\
\frac{1}{2}\left\|u_{m}\right\|_{2}^{2}+\left\|\nabla u_{m}\right\|_{2}^{2}+\left\|\nabla v_{m}\right\|_{2}^{2}+\left\|\dot{v}_{m}\right\|_{2}^{2} \\
+\left(\mu^{2}-\mu_{1}^{2}\right)\left\|v_{m}\right\|_{2}^{2}-A\left\|u_{m}\right\|_{2}^{B} .
\end{gathered}
$$

It is easy to see that

$$
\begin{aligned}
\left\|u_{m}\right\|_{p+1},\left\|v_{m}\right\|_{p+1}, & \left\|u_{m}\right\|_{H^{1}},\left\|v_{m}\right\|_{H^{1}} \\
& \leq C\left(\left\|\varphi_{m}\right\|_{2}^{2}+E\left(\varphi_{m}, \psi_{1 m}, \psi_{2 m}\right)+A\left\|\varphi_{m}\right\|_{2}^{B}\right)^{1 / 2},
\end{aligned}
$$

and

$$
\left\|\dot{v}_{m}\right\|_{2}^{2} \leq\left\|\varphi_{m}\right\|_{2}^{2}+E\left(\varphi_{m}, \psi_{1 m}, \psi_{2 m}\right)+A\left\|\varphi_{m}\right\|_{2}^{B}
$$


So

$\sup _{t}\left\|u_{m}\right\|_{X}, \sup _{t}\left\|v_{m}\right\|_{X}, \sup _{t}\left\|\dot{v}_{m}\right\|_{2} \leq M\left(\left\|\varphi_{m}\right\|_{2}, E\left(\varphi_{m}, \psi_{1 m}, \psi_{2 m}\right)\right)$.

CASE 2: $p+1>2^{*}$. From (1.4), we have

$\left\|\varphi_{m}\right\|_{2}^{2}+E\left(\varphi_{m}, \psi_{1 m}, \psi_{2 m}\right)$

$$
\begin{aligned}
\geq & \left\|u_{m}\right\|_{2}^{2}+\left\|\nabla u_{m}\right\|_{2}^{2}+\left\|\nabla v_{m}\right\|_{2}^{2}+\left\|\dot{v}_{m}\right\|_{2}^{2} \\
& +\left(\mu^{2}-\mu_{1}^{2}\right)\left\|v_{m}\right\|_{2}^{2}+C_{1}\left\|u_{m}\right\|_{p+1}^{p+1}+C_{1}\left\|v_{m}\right\|_{p+1}^{p+1},
\end{aligned}
$$

so

$$
\left\|u_{m}\right\|_{p+1},\left\|v_{m}\right\|_{p+1} \leq C\left(\left\|\varphi_{m}\right\|_{2}^{2}+E\left(\varphi_{m}, \psi_{1 m}, \psi_{2 m}\right)\right)^{1 /(p+1)} .
$$

Arguing as in Case 1, we also have

$$
\left\|u_{m}\right\|_{H^{1}},\left\|v_{m}\right\|_{H^{1}},\left\|\dot{v}_{m}\right\|_{2} \leq C\left(\left\|\varphi_{m}\right\|_{2}^{2}+E\left(\varphi_{m}, \psi_{1 m}, \psi_{2 m}\right)\right)^{1 / 2} .
$$

Combining the above two cases, we always have

$$
\sup _{t}\left\|u_{m}\right\|_{X}, \sup _{t}\left\|v_{m}\right\|_{X}, \sup _{t}\left\|\dot{v}_{m}\right\|_{2} \leq M\left(\left\|\varphi_{m}\right\|_{2}, E\left(\varphi_{m}, \psi_{1 m}, \psi_{2 m}\right)\right) .
$$

Since $\|\varphi\|_{2}$ and $E\left(\varphi, \psi_{1}, \psi_{2}\right)$ are continuous functions of $\varphi, \psi_{1}$ and $\psi_{2}$, by (2.11), we obtain

$$
\sup _{m} \sup _{t}\left\|u_{m}\right\|_{X}, \sup _{m} \sup _{t}\left\|v_{m}\right\|_{X}, \sup _{m} \sup _{t}\left\|\dot{v}_{m}\right\|_{2}<\infty
$$

i.e.,

$$
\sup _{m}\left\|u_{m}\right\|_{L^{\infty}(J, X)}, \sup _{m}\left\|v_{m}\right\|_{L^{\infty}(J, X)}, \sup _{m}\left\|\dot{v}_{m}\right\|_{L^{\infty}\left(J, L^{2}\right)}<\infty .
$$

Note that $f_{1}\left(\left|u_{m}\right|^{2}, v_{m}\right) u_{m}$ and $f_{2}\left(\left|u_{m}\right|^{2}, v_{m}\right)$ are bounded from $X \times X$ to $L^{2}+L^{(p+1) / p}$, and (2.16) implies that they are uniformly bounded in $L^{\infty}(J$, $L^{2}+L^{(p+1) / p}$ ) and therefore $\xi_{m}, \eta_{m}$ defined by

$$
\left\{\begin{array}{l}
\xi_{m}=-\Delta u_{m}+f_{1}\left(\left|u_{m}\right|^{2}, v_{m}\right) u_{m}, \\
\eta_{m}=\Delta v_{m}-\mu^{2} v_{m}-\frac{1}{2} f_{2}\left(\left|u_{m}\right|^{2}, v_{m}\right),
\end{array}\right.
$$

are uniformly bounded in $L^{\infty}\left(J, X^{\prime}\right)$. It follows from the relation

$$
\begin{aligned}
\left\|u_{m}(t)-u_{m}(s)\right\|_{2}^{2} & =-2 \int_{s}^{t} \operatorname{Im}\left(u_{m}(s), \xi_{m}(\tau)\right) d \tau \\
& \leq 2|s-t|\left\|u_{m}\right\|_{L^{\infty}(J, X)}\left\|\xi_{m}\right\|_{L^{\infty}\left(J, X^{\prime}\right)}
\end{aligned}
$$

that the sequence $\left\{u_{m}\right\}$ is uniformly (in $m$ and $t$ ) Hölder continuous in $L^{2}$ with exponent $1 / 2$, i.e., $u_{m}(t) \in \mathcal{C}^{1 / 2}\left(J, L^{2}\right)$. From this together with the fact that $u_{m} \in L^{\infty}(J, X)$, we obtain by interpolation

$$
u_{m}(t) \in \bigcap_{2 \leq r<\max \left(p+1,2^{*}\right)} \mathcal{C}^{\alpha(r)}\left(J, L^{r}\right) .
$$


Note that $v_{m}(t) \in L^{\infty}(J, X)$ and $\left(\Delta v_{m}-\mu^{2} v_{m}\right)-\frac{1}{2} f_{2}\left(\left|u_{m}\right|^{2}, v_{m}\right) \in L^{\infty}\left(J, X^{\prime}\right)$ $\hookrightarrow \mathcal{D}^{\prime}\left(J, X^{\prime}\right)$. By a similar argument to Lemma $2.1(2)$, we obtain

$$
\dot{v}_{m} \in \mathcal{C}^{L}\left(J, X^{\prime}\right) \cap \mathcal{C}_{w}\left(J, L^{2}\right) \cap L^{\infty}\left(J, L^{2}\right),
$$

and

$$
v_{m} \in \mathcal{C}_{w}(J, X) \cap \mathcal{C}^{L}\left(J, L^{2}\right) \cap \bigcap_{2<r<\max \left(p+1,2^{*}\right)} \mathcal{C}^{\beta(r)}\left(J, L^{r}\right) .
$$

STEP 3: Convergence of subsequences. Noting that $\left(L^{\infty}(J, X)\right)^{\prime}=$ $L^{1}\left(J, X^{\prime}\right),(2.15)$ means one can extract subsequences that weak* converge to $u, v$ and $\dot{v}$ respectively, still called $\left\{u_{m}\right\},\left\{v_{m}\right\}$ and $\left\{\dot{v}_{m}\right\}$ for simplicity, i.e., as $m \rightarrow \infty$,

$$
\begin{cases}u_{m} \stackrel{w^{*}}{\rightarrow} u & \text { in } L^{\infty}(J, X), \\ v_{m} \stackrel{w^{*}}{\rightarrow} v & \text { in } L^{\infty}(J, X), \\ \dot{v}_{m} \stackrel{w^{*}}{\rightarrow} \dot{v} & \text { in } L^{\infty}\left(J, L^{2}\right) .\end{cases}
$$

To prove that $(u, v)$ satisfies (1.1), we need some preparations. Let

$$
\widetilde{u}=i \dot{u}+\Delta u, \quad \widetilde{v}=\ddot{v}-\Delta v+\mu^{2} v .
$$

It is easy to see $\widetilde{u}, \widetilde{v} \in \mathcal{D}^{\prime}(J, X)+L^{\infty}\left(J, H^{-1}\right)$. We first prove that

$$
\begin{array}{ll}
f_{1}\left(\left|u_{m}\right|^{2}, v_{m}\right) u_{m} \stackrel{w^{*}}{\rightarrow} \widetilde{u} & \text { in } L^{\infty}\left(J, L^{2}+L^{(p+1) / p}\right), \\
-\frac{1}{2} f_{2}\left(\left|u_{m}\right|^{2}, v_{m}\right) \stackrel{w^{*}}{\rightarrow} \widetilde{v} & \text { in } L^{\infty}\left(J, L^{2}+L^{(p+1) / p}\right) .
\end{array}
$$

By (2.15), (H1') and Lemma 2.1, $\left\{f_{1}\left(\left|u_{m}\right|^{2}, v_{m}\right) u_{m}\right\},\left\{f_{2}\left(\left|u_{m}\right|^{2}, v_{m}\right)\right\}$ are bounded and therefore weak* relatively compact in $L^{\infty}\left(J, L^{2}+L^{(p+1) / p}\right)$. On the other hand, from $(2.10)$ and $(2.19)$, for all $\theta(t) \in \mathcal{C}_{\mathrm{c}}^{\infty}(J, \mathbb{C})$ and $j \leq m$,

$$
\begin{gathered}
\int_{J}\left(w_{j}, f_{1}\left(\left|u_{m}\right|^{2}, v_{m}\right) u_{m}\right) \theta(\tau) d \tau=\int_{J}\left(w_{j}, i \dot{u}_{m}+\Delta u_{m}\right) \theta(\tau) d \tau \\
=-\int_{J}\left(\left(w_{j}, i u_{m}\right) \dot{\theta}(\tau)-\left(w_{j}, \Delta u_{m}\right) \theta(\tau)\right) d \tau \\
\stackrel{m \rightarrow \infty}{\longrightarrow}-\int_{J}\left(\left(w_{j}, i u\right) \dot{\theta}(\tau)-\left(w_{j}, \Delta u\right) \theta(\tau)\right) d \tau \\
=\int_{J}\left(w_{j}, i \dot{u}+\Delta u\right) \theta(\tau) d \tau=\int_{J}\left(w_{j}, \widetilde{u}\right) \theta(\tau) d \tau
\end{gathered}
$$

Since $\left\{w_{j}\right\}$ is a basis of $X$, and $\bar{\theta}(\tau) w_{j} \in \mathcal{C}_{\mathrm{c}}^{\infty}(J, X),(2.22)$ means that any weak* convergent subsequence of $\left\{f_{1}\left(\left|u_{m}\right|^{2}, v_{m}\right) u_{m}\right\}$ in $L^{\infty}\left(J, L^{2}+L^{(p+1) / p}\right)$ converges to $\widetilde{u}$ in $\mathcal{D}^{\prime}\left(J, X^{\prime}\right)$, therefore $(2.20)$ holds. 
As to $v_{m}$, by $(2.19)$, for all $\theta(t) \in \mathcal{C}_{\mathrm{c}}^{\infty}(J, \mathbb{C})$ and $j \leq m$,

$$
\begin{aligned}
\int_{J}\left(w_{j},-\frac{1}{2} f_{2}\left(\left|u_{m}\right|^{2}, v_{m}\right)\right) \theta(\tau) d \tau=\int_{J}\left(w_{j}, \ddot{v}_{m}-\Delta v_{m}+\mu^{2} v_{m}\right) \theta(\tau) d \tau \\
=\int_{J}\left(\left(w_{j}, v_{m}\right) \ddot{\theta}(\tau)+\left(w_{j},\left(\mu^{2}-\Delta\right) v_{m}\right) \theta(\tau)\right) d \tau \\
\stackrel{m \rightarrow \infty}{\longrightarrow} \int_{J}\left(\left(w_{j}, v\right) \ddot{\theta}(\tau)+\left(w_{j},\left(\mu^{2}-\Delta\right) v\right) \theta(\tau)\right) d \tau \\
=\int_{J}\left(w_{j}, \ddot{v}-\Delta v+\mu^{2} v\right) \theta(\tau) d \tau=\int_{J}\left(w_{j}, \widetilde{v}\right) \theta(\tau) d \tau
\end{aligned}
$$

therefore (2.21) holds.

By definition of $\widetilde{u}$ and $\widetilde{v}$,

$$
\left\{\begin{array}{l}
\dot{u}(t)=-i \widetilde{u}+i \Delta u \in L^{\infty}\left(J, L^{(p+1) / p}+H^{-1}\right)=L^{\infty}\left(J, X^{\prime}\right), \\
\ddot{v}(t)=\widetilde{v}+\Delta v-\mu^{2} v \in L^{\infty}\left(J, L^{(p+1) / p}+H^{-1}\right)=L^{\infty}\left(J, X^{\prime}\right) .
\end{array}\right.
$$

From this together with $u(t), v(t) \in L^{\infty}(J, X)$, by a similar argument to that in Lemma 2.1(2), we have

$$
\left\{\begin{array}{l}
u(t) \in \mathcal{C}_{w}(J, X) \cap \bigcap_{2 \leq r<\max \left(p+1,2^{*}\right)} \mathcal{C}^{\alpha(r)}\left(J, L^{r}\right) \\
v(t) \in \mathcal{C}_{w}(J, X) \cap \mathcal{C}^{L}\left(J, L^{2}\right) \cap \bigcap_{2<r<\max \left(p+1,2^{*}\right)} \mathcal{C}^{\beta(r)}\left(J, L^{r}\right) \\
\dot{v}(t) \in \mathcal{C}_{w}\left(J, L^{2}\right) \cap \mathcal{C}^{L}\left(J, X^{\prime}\right)
\end{array}\right.
$$

We next prove that for all $t \in J$,

$$
u_{m}(t) \stackrel{w}{\rightarrow} u(t), \quad v_{m}(t) \stackrel{w}{\rightarrow} v(t)
$$

Firstly, for fixed $t,(2.15)$ implies that $\left\{u_{m}(t)\right\}$ is bounded on $X$ uniformly in $m$, and is therefore weakly relatively compact since $X$ is reflexive. By (2.19), we only need to prove that it has the unique weak limit point $u$. Suppose that it has another limit point $\chi$ in $X$. For $\tau$ in a neighborhood of $t,(2.17)$ and (2.23) imply

$$
\|u(\tau)-u(t)\|_{2},\left\|u_{m}(\tau)-u_{m}(t)\right\|_{2} \leq C|t-\tau|^{1 / 2}
$$

so for $\gamma>0$ we can estimate

$$
\begin{aligned}
\| u(t) & -\chi(t) \|_{2}^{2}=\left(u(t)-\chi(t), u_{m}(t)-\chi(t)\right) \\
& +\frac{1}{2 \gamma} \int_{t-\gamma}^{t+\gamma}\left(u(t)-\chi(t), u(t)-u(\tau)+u_{m}(\tau)-u_{m}(t)+u(\tau)-u_{m}(\tau)\right) d \tau
\end{aligned}
$$




$$
\begin{aligned}
& \leq\left(u(t)-\chi(t), u_{m}(t)-\chi(t)\right)+2 C \gamma^{1 / 2}\|u(t)-\chi(t)\|_{2} \\
& \quad+\frac{1}{2 \gamma} \int_{t-\gamma}^{t+\gamma}\left(u(t)-\chi(t), u(\tau)-u_{m}(\tau)\right) d \tau \\
& =I+I I+I I I .
\end{aligned}
$$

Now $u_{m}(t) \stackrel{w}{\rightarrow} \chi$ in $X$ implies $I=0, u_{m}(t) \stackrel{w^{*}}{\rightarrow} u(t)$ in $L^{\infty}(J, X)$ implies $I I I=0$ for fixed $\gamma, I I$ tends to zero with $\gamma$, so $\chi(t)=u(t)$ in this interval. Iterating this process, we obtain $\chi(t)=u(t)$ in the whole interval $J$.

The same result for $\left\{v_{m}\right\}$ can be deduced from (2.18) and (2.23).

STEP 4: Initial conditions. For all $\theta(t) \in \mathcal{C}_{\mathrm{c}}^{\infty}(J, \mathbb{C})$ with $\theta(0)=1$. Integrating by parts, we have for $J_{0}=(0, \infty) \cap J$,

$$
\int_{J_{0}}\left(w_{j}, i u_{m}(\tau) \dot{\theta}(\tau)+\xi_{m}(\tau) \theta(\tau)\right) d \tau=-\left(w_{j}, i \varphi_{m}\right) .
$$

Letting $m \rightarrow \infty$ and using (2.11), we get

i.e.,

$$
\int_{J_{0}}(i u(\tau) \dot{\theta}(\tau)+i \dot{u}(\tau) \theta(\tau)) d \tau=-i \varphi
$$

$$
\left.i u(\tau) \theta(\tau)\right|_{J_{0}}=-i \varphi \theta(0)
$$

so $u(0)=\varphi$.

For $v_{m}$, we can prove similarly that $v(0)=\psi_{1}$ and $\dot{v}(0)=\psi_{2}$.

STEP 5: $(u, v)$ satisfies (1.1). By definition of $\widetilde{u}, \widetilde{v}$, we only need to prove that

$$
\widetilde{u}=f_{1}\left(|u|^{2}, v\right) u, \quad \widetilde{v}=-\frac{1}{2} f_{2}\left(|u|^{2}, v\right) .
$$

(2.20) and (2.21) imply, for $J$ and any bounded open set $\Omega \subset \mathbb{R}^{n}$,

$$
\left\|f_{1}\left(\left|u_{m}\right|^{2}, v_{m}\right) u_{m}\right\|_{(p+1) / p, J \times \Omega},\left\|f_{2}\left(\left|u_{m}\right|^{2}, v_{m}\right)\right\|_{(p+1) / p, J \times \Omega}<\infty,
$$

So

$$
f_{1}\left(\left|u_{m}\right|^{2}, v_{m}\right) u_{m} \stackrel{w}{\rightarrow} \widetilde{u}, \quad f_{2}\left(\left|u_{m}\right|^{2}, v_{m}\right) \stackrel{w}{\rightarrow} \widetilde{v}
$$

in $L^{(p+1) / p}(J \times \Omega)$.

On the other hand, $u_{m}(t) \in \mathcal{C}^{1 / 2}\left(J, L^{2}\right)$ implies $u_{m}$ is equicontinuous in $J \times \Omega$, and (2.15) implies $u_{m}$ is uniformly bounded in $H^{1}(\Omega)$, so that, by $H^{1}(\Omega) \hookrightarrow \hookrightarrow L^{2}(\Omega)$ and Ascoli's theorem, the sequence $\left\{u_{m}\right\}$ is relatively compact in $\mathcal{C}\left(J, L^{2}(\Omega)\right)$. Since $(2.19)$ means $u_{m} \stackrel{w^{*}}{\rightarrow} u$ in $L^{\infty}\left(J, L^{2}(\Omega)\right)$, we have

$$
u_{m} \rightarrow u \quad \text { in } \mathcal{C}\left(J, L^{2}(\Omega)\right)
$$

thus

$$
u_{m} \rightarrow u \quad \text { in } L^{2}(J \times \Omega)
$$


Moreover, $v_{m} \in \mathcal{C}^{L}\left(J, L^{2}\right)$ (see (2.18)) implies $v_{m}$ is equicontinuous in $J \times \Omega$, and by a similar argument, we also have

$$
v_{m} \rightarrow v \quad \text { in } \mathcal{C}\left(J, L^{2}(\Omega)\right)
$$

thus

$$
v_{m} \rightarrow v \quad \text { in } L^{2}(J \times \Omega) .
$$

We can then extract subsequences of $\left\{u_{m}\right\}$ and $\left\{v_{m}\right\}$ such that

$$
u_{m_{k}} \stackrel{\text { a.e. }}{\rightarrow} u, \quad v_{m_{k}} \stackrel{\text { a.e. }}{\rightarrow} v
$$

and

$$
\left\{\begin{array}{l}
f_{1}\left(\left|u_{m_{k}}\right|^{2}, v_{m_{k}}\right) u_{m_{k}} \stackrel{\text { a.e. }}{\rightarrow} f_{1}\left(|u|^{2}, v\right) u \\
f_{2}\left(\left|u_{m_{k}}\right|^{2}, v_{m_{k}}\right) \stackrel{\text { a.e. }}{\rightarrow} f_{2}\left(|u|^{2}, v\right)
\end{array}\right.
$$

in $J \times \Omega$. Moreover, $f_{1}\left(\left|u_{m}\right|^{2}, v_{m}\right) u_{m}, f_{2}\left(\left|u_{m}\right|^{2}, v_{m}\right) \in L^{\infty}\left(J, L^{2}+L^{(p+1) / p}\right)$ implies $f_{1}\left(\left|u_{m_{k}}\right|^{2}, v_{m_{k}}\right) u_{m_{k}}, f_{2}\left(\left|u_{m_{k}}\right|^{2}, v_{m_{k}}\right) \in L^{(p+1) / p}(J \times \Omega)$, and then

$$
\left\{\begin{array}{l}
f_{1}\left(\left|u_{m_{k}}\right|^{2}, v_{m_{k}}\right) u_{m_{k}} \stackrel{w}{\rightarrow} f_{1}\left(|u|^{2}, v\right) u \\
-\frac{1}{2} f_{2}\left(\left|u_{m_{k}}\right|^{2}, v_{m_{k}}\right) \stackrel{w}{\rightarrow}-\frac{1}{2} f_{2}\left(|u|^{2}, v\right)
\end{array}\right.
$$

in $L^{(p+1) / p}(J \times \Omega)$. From (2.25) and (2.26), (2.24) holds in $J \times \Omega$ because the weak limit points of $\left\{u_{m}\right\}$ and $\left\{v_{m}\right\}$ are both unique.

Since $\Omega$ is arbitrary, we obtain, for all $(t, x) \in J \times \mathbb{R}^{n}$,

$$
f_{1}\left(|u|^{2}, v\right) u=\widetilde{u}, \quad-\frac{1}{2} f_{2}\left(|u|^{2}, v\right)=\widetilde{v}
$$

Step 6: Conservation laws. Since $(u, v)$ satisfies (1.1), $\|u(t)\|_{2}=\|\varphi\|_{2}$ follows from Lemma 2.1(4). In order to prove the energy inequality, we need some properties.

For fixed $t \in J$, since $X$ is reflexive, the convergences

as $m \rightarrow \infty$, imply

$$
\begin{cases}u_{m} \stackrel{w^{*}}{\rightarrow} u & \text { in } X \\ v_{m} \stackrel{w^{*}}{\rightarrow} v & \text { in } X \\ \dot{v}_{m} \stackrel{w^{*}}{\rightarrow} \dot{v} & \text { in } L^{2}\end{cases}
$$

$$
\begin{cases}u_{m} \stackrel{w}{\stackrel{w}{\rightarrow}} u & \text { in } L^{2}, \\ v_{m} \stackrel{w}{\rightarrow} v & \text { in } L^{2} \\ \dot{v}_{m} \stackrel{w}{\rightarrow} \dot{v} & \text { in } L^{2} .\end{cases}
$$

On the other hand, by the conservation of $L^{2}$-norm,

$$
\lim _{m \rightarrow \infty}\left\|u_{m}(t)\right\|_{2}=\lim _{m \rightarrow \infty}\left\|\varphi_{m}\right\|_{2}=\|\varphi\|_{2}=\|u(t)\|_{2},
$$

thus

$$
\lim _{m \rightarrow \infty}\left\|u_{m}(t)-u(t)\right\|_{2}=0 .
$$

Moreover, $u_{m}(t) \in \mathcal{C}^{1 / 2}\left(J, L^{2}\right)$ means $u_{m}$ is equicontinuous in $L^{2}$, so $u_{m}(t)$ is uniformly strongly convergent in $L^{2}$ for all $t \in J$. 
By the interpolation inequality

$$
\left\|u_{m}(t)-u(t)\right\|_{r} \leq C\left(\left\|u_{m}\right\|_{X}^{1-\theta}+\|u\|_{X}^{1-\theta}\right)\left\|u_{m}-u\right\|_{2}^{\theta},
$$

we get

$$
\lim _{m \rightarrow \infty}\left\|u_{m}-u\right\|_{\mathcal{C}\left(J, L^{r}\right)}=0
$$

for all $2 \leq r<\max \left(p+1,2^{*}\right)$.

Further, (2.18) means $v_{m}$ is equicontinuous in $J$, and $\sup _{m} \sup _{t}\left\|v_{m}\right\|_{X}<$ $\infty$ means $\sup _{m} \sup _{t}\left\|v_{m}\right\|_{L^{2}}<\infty$. By Ascoli's theorem, $\left\{v_{m}(t)\right\}$ is relatively compact in $\mathcal{C}\left(J, L^{2}\right)$. Note that $v_{m} \stackrel{w^{*}}{\rightarrow} v$ in $L^{\infty}(J, X)$ implies $v_{m} \stackrel{w^{*}}{\rightarrow} v$ in $L^{\infty}\left(J, L^{2}\right)$, so we obtain

$$
v_{m} \rightarrow v \quad \text { in } \mathcal{C}\left(J, L^{2}\right)
$$

By interpolation, we also have

$$
\lim _{m \rightarrow \infty}\left\|v_{m}-v\right\|_{\mathcal{C}\left(J, L^{r}\right)}=0
$$

We have proved in Step 1 that $E\left(u_{m}, v_{m}, \dot{v}_{m}\right)=E\left(\varphi_{m}, \psi_{1 m}, \psi_{2 m}\right)$. Letting $m \rightarrow \infty$, we have from $(2.11)$

$$
\begin{aligned}
&\left\|\nabla \varphi_{m}\right\|_{2}^{2}+\left\|\nabla \psi_{1 m}\right\|_{2}^{2}+\left\|\underset{\psi_{2 m}}{ }\right\|_{2}^{2}+\mu^{2}\left\|\psi_{1 m}\right\|_{2}^{2} \\
& \stackrel{m \rightarrow \infty}{\longrightarrow}\|\nabla \varphi\|_{2}^{2}+\left\|\nabla \psi_{1}\right\|_{2}^{2}+\left\|\psi_{2}\right\|_{2}^{2}+\mu^{2}\left\|\psi_{1}\right\|_{2}^{2},
\end{aligned}
$$

and

$$
\begin{aligned}
& \quad\left|\int_{\mathbb{R}^{n}} F\left(\left|\varphi_{m}\right|^{2}, \psi_{1 m}\right) d x-\int_{\mathbb{R}^{n}} F\left(|\varphi|^{2}, \psi_{1}\right) d x\right| \\
& \leq \int_{\mathbb{R}^{n}}\left(\left|F\left(\left|\varphi_{m}\right|^{2}, \psi_{1 m}\right)-F\left(|\varphi|^{2}, \psi_{1 m}\right)\right|+\left|F\left(|\varphi|^{2}, \psi_{1 m}\right)-F\left(|\varphi|^{2}, \psi_{1}\right)\right|\right) d x
\end{aligned}
$$$$
\left.\leq \int_{\mathbb{R}^{n}} \int_{0}^{1} \mid f_{1}\left(\alpha\left|\varphi_{m}\right|^{2}+(1-\alpha)|\varphi|^{2}, \psi_{1 m}\right)\right)\left(\left|\varphi_{m}\right|+|\varphi|\right)||\left|\varphi_{m}\right|-|\varphi| \mid d \alpha d x
$$

$$
+\int_{\mathbb{R}^{n}}^{1}\left|f_{0}^{1}\right| f_{2}\left(|\varphi|^{2}, \beta \psi_{1 m}+(1-\beta) \psi_{1}\right)|| \psi_{1 m}-\psi_{1} \mid d \beta d x
$$

$$
=I+I I \stackrel{m \rightarrow \infty}{\longrightarrow} 0 .
$$

In fact, we again decompose $f_{1}$ and $f_{2}$ as in (2.4)-(2.6), to get

$$
\begin{aligned}
I \leq & C \int_{\mathbb{R}^{n}}\left(\left|\varphi_{m}\right|^{p}+|\varphi|^{p}+\left|\psi_{1 m}\right|^{p}\right)\left|\varphi_{m}-\varphi\right| d x \\
& +C \int_{\mathbb{R}^{n}}\left(\left|\varphi_{m}\right|+|\varphi|\right)\left|\varphi_{m}-\varphi\right| d x \\
\leq & C\left(\left\|\left|\varphi_{m}\right|^{p}\right\|_{(p+1) / p}+\left\||\varphi|^{p}\right\|_{(p+1) / p}+\left\|\left|\psi_{1 m}\right|^{p}\right\|_{(p+1) / p}\right)\left\|\varphi_{m}-\varphi\right\|_{X} \\
& +C\left(\left\|\varphi_{m}\right\|_{2}+\|\varphi\|_{2}\right)\left\|\varphi_{m}-\varphi\right\|_{2} \\
\leq & C\left\|\varphi_{m}-\varphi\right\|_{X} \stackrel{m \rightarrow \infty}{\longrightarrow} 0,
\end{aligned}
$$


and similarly

$$
\begin{aligned}
I I \leq & C \int_{\mathbb{R}^{n}}\left(|\varphi|^{p}+\left|\psi_{1 m}\right|^{p}+\left|\psi_{1}\right|^{p}\right)\left|\psi_{1 m}-\psi_{1}\right| d x \\
& +C \int_{\mathbb{R}^{n}}\left(|\varphi|+\left|\psi_{1 m}\right|+\left|\psi_{1}\right|\right)\left|\psi_{1 m}-\psi_{1}\right| d x \\
\leq & C\left\|\psi_{1 m}-\psi_{1}\right\|_{X} \stackrel{m \rightarrow \infty}{\longrightarrow} 0 .
\end{aligned}
$$

Thus

$$
E\left(\varphi_{m}, \psi_{1 m}, \psi_{2 m}\right) \stackrel{m \rightarrow \infty}{\longrightarrow} E\left(\varphi, \psi_{1}, \psi_{2}\right)
$$

On the other hand,

$$
\begin{aligned}
E\left(u_{m}, v_{m}, \dot{v}_{m}\right)= & \left\|\nabla u_{m}\right\|_{2}^{2}+\left\|\nabla v_{m}\right\|_{2}^{2}+\left\|\dot{v}_{m}\right\|_{2}^{2}+\mu^{2}\left\|v_{m}\right\|_{2}^{2} \\
& +\int_{\mathbb{R}^{n}} F\left(\left|u_{m}\right|^{2}, v_{m}\right) d x
\end{aligned}
$$

CAsE 1: $p+1<2^{*}$. By the same process as in the proof of $(2.27)$, we obtain

$$
\int_{\mathbb{R}^{n}} F\left(\left|u_{m}\right|^{2}, v_{m}\right) d x \stackrel{m \rightarrow \infty}{\longrightarrow} \int_{\mathbb{R}^{n}} F\left(|u|^{2}, v\right) d x,
$$

and since $\left\|\nabla u_{m}\right\|_{2}^{2},\left\|\nabla v_{m}\right\|_{2}^{2},\left\|\dot{v}_{m}\right\|_{2}^{2},\left\|v_{m}\right\|_{2}^{2}$ are all weakly lower semicontinuous, we get

$$
\liminf _{m \rightarrow \infty} E\left(u_{m}, v_{m}, \dot{v}_{m}\right) \geq E(u, v, \dot{v})
$$

so (1.7) holds.

CASE $2: p+1 \geq 2^{*}$. Using $F_{1}$ instead of $F$, and $\bar{p}$ instead of $p$ in $\left(\mathrm{H} 1^{\prime}\right)$, we can still prove that

$$
\int_{\mathbb{R}^{n}} F_{1}\left(\left|u_{m}\right|^{2}, v_{m}\right) d x \stackrel{m \rightarrow \infty}{\longrightarrow} \int_{\mathbb{R}^{n}} F_{1}\left(|u|^{2}, v\right) d x
$$

and since $\left\|\nabla u_{m}\right\|_{2}^{2},\left\|\nabla v_{m}\right\|_{2}^{2},\left\|\dot{v}_{m}\right\|_{2}^{2},\left\|v_{m}\right\|_{2}^{2}, \int_{\mathbb{R}^{n}} F_{2}\left(\left|u_{m}\right|^{2}, v_{m}\right) d x$ are all weakly lower semicontinuous, we also have (1.7).

3. Uniqueness of solutions. In this section, we will prove that under stronger assumptions on the interactions, the solutions obtained in $\S 2$ are unique. Clearly (H3) implies (H1), and $p<1+4 /(n-2)$ implies that $H^{1} \hookrightarrow$ $L^{p+1}$, so that $X=H^{1}$. Firstly, let us introduce some preliminary lemmas.

LEMMA 3.1. Let $n \geq 3$, @ and $r$ satisfy

$$
\varrho \leq 1-\beta(r), \quad 0 \leq \delta(r) \leq n / 2, \quad 0 \leq \delta(r)+\varrho-1<1 / 2,
$$

and let $q$ satisfy

$$
\begin{gathered}
\delta(r)+\varrho-1=1 / q \\
\delta(r)+\varrho-1 \leq 2 / q \leq \delta(r), \quad q>2 .
\end{gathered}
$$


Then for any $\left(\varphi, \psi_{1}, \psi_{2}\right) \in H^{1} \times H^{1} \times L^{2}$,

$$
\left\{\begin{array}{l}
\|U(t) \varphi\|_{L^{q}\left(\mathbb{R}, B_{r, 2}^{o}\right)} \leq C\|\varphi\|_{H^{1}}, \\
\left\|K(t) \psi_{2}+\dot{K}(t) \psi_{1}\right\|_{L^{q}\left(\mathbb{R}, B_{r, 2}^{\varrho}\right)} \leq C\left(\left\|\psi_{2}\right\|_{2}+\left\|\psi_{1}\right\|_{H^{1}}\right) .
\end{array}\right.
$$

The proof is given in $[10,11]$.

LEMMA 3.2. Let $f_{1}\left(|u|^{2}, v\right), f_{2}\left(|u|^{2}, v\right)$ satisfy (H3) for $1 \leq p<\infty$, and let $0<\lambda<1,1 \leq l^{\prime} \leq k \leq \infty, 1 \leq m \leq \infty$ and $1 / s=1 / l^{\prime}-1 / k$. Then

$$
\begin{aligned}
\left\|f_{1}\left(|u|^{2}, v\right) u\right\|_{B_{l^{\prime}, m}^{\lambda}} & \leq C\left(\|u\|_{B_{k, m}^{\lambda}}+\|v\|_{B_{k, m}^{\lambda}}\right)\left(\|u\|_{(p-1) s}^{p-1}+\|v\|_{(p-1) s}^{p-1}\right), \\
\left\|f_{2}\left(|u|^{2}, v\right)\right\|_{B_{l^{\prime}, m}^{\lambda}} & \leq C\left(\|u\|_{B_{k, m}^{\lambda}}+\|v\|_{B_{k, m}^{\lambda}}\right)\left(\|u\|_{(p-1) s}^{p-1}+\|v\|_{(p-1) s}^{p-1}\right) .
\end{aligned}
$$

Proof. Use the equivalent norm of the Besov space $B_{l^{\prime}, m}^{\lambda}$,

$$
\|v\|_{B_{l^{\prime}, m}^{\lambda}}=\|v\|_{l^{\prime}}+\left\{\int_{0}^{\infty}\left(\sup _{|y| \leq t} t^{-\lambda}\left\|\tau_{y} v-v\right\|_{l^{\prime}}\right)^{m} \frac{d t}{t}\right\}^{1 / m},
$$

where $\tau_{y}$ denotes the space translation by $y \in \mathbb{R}^{n}$.

We only need to prove the result for $\dot{B}_{p, q}^{\sigma}$ instead of $B_{p, q}^{\sigma}$. From (H3) and the Hölder inequality, we have

$$
\left\{\begin{array}{l}
\left\|f_{1}\left(|u(x+y)|^{2}, v(x+y)\right) u(x+y)-f_{1}\left(|u(x)|^{2}, v(x)\right) u(x)\right\|_{l^{\prime}} \\
\quad \leq C\left(\|u\|_{(p-1) s}^{p-1}+\|v\|_{(p-1) s}^{p-1}\right)\left(\|u(x+y)-u(x)\|_{k}+\|v(x+y)-v(x)\|_{k}\right), \\
\left\|f_{2}\left(|u(x+y)|^{2}, v(x+y)\right)-f_{2}\left(|u(x)|^{2}, v(x)\right)\right\|_{l^{\prime}} \\
\leq C\left(\|u\|_{(p-1) s}^{p-1}+\|v\|_{(p-1) s}^{p-1}\right)\left(\|u(x+y)-u(x)\|_{k}+\|v(x+y)-v(x)\|_{k}\right),
\end{array}\right.
$$

from which (3.5) follows immediately.

LEMMA 3.3. Let $n \geq 3,0 \in I$ be an open interval, and $\left(\varphi, \psi_{1}, \psi_{2}\right) \in$ $H^{1} \times H^{1} \times L^{2}$. If $\varrho, r$ satisfy (3.2) of Lemma 3.1, and $1 / q \geq \varrho+\delta(r)-1$, then $u(t), v(t) \in L_{\mathrm{loc}}^{q}\left(I, B_{r, 2}^{\varrho}\right)$ and for any compact $J \subset I$ containing 0 ,

$$
\begin{aligned}
& \|u\|_{L^{q}\left(J, B_{r, 2}^{\varrho}\right)},\|v\|_{L^{q}\left(J, B_{r, 2}^{\varrho}\right)} \\
& \quad \leq C\left(\|u\|_{H^{1}},\|v\|_{H^{1}},\|\varphi\|_{H^{1}},\left\|\psi_{1}\right\|_{H^{1}},\left\|\psi_{2}\right\|_{L^{2}}\right) .
\end{aligned}
$$

Proof. Consider

$$
\left\{\begin{array}{l}
u(t)=U(t) \varphi-i \int_{0}^{t} U(t-\tau) f_{1}\left(|u|^{2}, v\right) u(\tau) d \tau \\
v(t)=\dot{K}(t) \psi_{1}+K(t) \psi_{2}-\frac{1}{2} \int_{0}^{t} K(t-\tau) f_{2}\left(|u|^{2}, v\right) d \tau
\end{array}\right.
$$

Let $\sigma=\varrho+\delta(r)-1$, and choose $r, \sigma^{\prime}$ and $\sigma^{\prime \prime}$ such that 


$$
1 \leq \delta(r) \leq n / 2, \quad 0 \leq \sigma^{\prime}<\sigma^{\prime \prime} \leq \sigma^{\prime}+\varepsilon<1 / 2,
$$

with $\varepsilon$ to be chosen later.

Claim.

$$
\begin{gathered}
\left\|U(t) f_{1}\left(|u|^{2}, v\right) u\right\|_{B_{r, 2}^{e^{\prime \prime}}} \leq C|t|^{-\delta(l)}\left(1+\|u\|_{B_{r, 2}^{e^{\prime}}}^{\nu}+\|v\|_{B_{r, 2}^{e^{\prime}}}^{\nu}\right), \\
\left\|K(t) f_{2}\left(|u|^{2}, v\right)\right\|_{B_{r, 2}^{e^{\prime \prime}}} \leq C|t|^{-\gamma(l)}\left(1+\|u\|_{B_{r, 2}^{e^{\prime}}}^{\nu}+\|v\|_{B_{r, 2}^{e^{\prime}}}^{\nu}\right)
\end{gathered}
$$

for $0 \leq \gamma<1,0 \leq \delta<1,0 \leq \nu \leq 1$ and $C$ depending only on $\gamma, \nu$ and $\varepsilon$, but not on $\varrho^{\prime}$ and $\varrho^{\prime \prime}$.

By an $L^{r}-L^{r^{\prime}}$ estimate and Sobolev embedding, we have

$$
\begin{aligned}
\left\|U(t) f_{1}\left(|u|^{2}, v\right) u\right\|_{B_{r, 2}^{e^{\prime \prime}}} & \leq C\left\|U(t) f_{1}\left(|u|^{2}, v\right) u\right\|_{B_{l, 2}^{\lambda+1-2 \beta(l)}} \\
& \leq C|t|^{-\delta(l)}\left\|f_{1}\left(|u|^{2}, v\right) u\right\|_{B_{l^{\prime}, 2}^{\lambda+1-2 \beta(l)}} \\
& \leq C|t|^{-\delta(l)}\left\|f_{1}\left(|u|^{2}, v\right) u\right\|_{B_{l^{\prime}, 2}^{\lambda}}
\end{aligned}
$$

and

$$
\begin{aligned}
\left\|K(t) f_{2}\left(|u|^{2}, v\right)\right\|_{B_{r, 2}^{e^{\prime \prime}}} & \leq C\left\|K(t) f_{2}\left(|u|^{2}, v\right)\right\|_{B_{l, 2}^{\lambda+1-2 \beta(l)}} \\
& \leq C|t|^{-\gamma(l)}\left\|f_{2}\left(|u|^{2}, v\right)\right\|_{B_{l^{\prime}, 2}^{\lambda}}
\end{aligned}
$$

where $B_{l, 2}^{\lambda+1-2 \beta(l)} \hookrightarrow B_{r, 2}^{\varrho^{\prime \prime}}$ is ensured by the condition

$$
\frac{1}{l}-\frac{1}{r} \leq \frac{\lambda+1-2 \beta(l)-\varrho^{\prime \prime}}{n} \quad \text { for } r \geq l,
$$

therefore by

$$
\lambda \geq \sigma^{\prime \prime}+\frac{\delta(l)}{n},
$$

in particular by

$$
\lambda=\sigma^{\prime \prime}+\frac{\delta(l)}{n} .
$$

And $B_{l^{\prime}, 2}^{\lambda} \hookrightarrow B_{l^{\prime}, 2}^{\lambda+1-2 \beta(l)}$ is ensured by the condition

$$
\lambda \geq \lambda+1-2 \beta(l),
$$

hence by

$$
\beta(l) \geq 1 / 2 .
$$

Noting that $0<\sigma^{\prime \prime}<1 / 2$, we have $0<\lambda<1$ for $2 \leq l \leq \infty$, and using Lemma 3.2 for $m=2$, we obtain

$$
\left\{\begin{array}{l}
\left\|f_{1}\left(|u|^{2}, v\right) u\right\|_{B_{l^{\prime}, 2}^{\lambda}} \leq C\left(\|u\|_{B_{k, 2}^{\lambda}}+\|v\|_{B_{k, 2}^{\lambda}}\right)\left(\|u\|_{(p-1) s}^{p-1}+\|v\|_{(p-1) s}^{p-1}\right), \\
\left\|f_{2}\left(|u|^{2}, v\right)\right\|_{B_{l^{\prime}, 2}^{\lambda}}^{p} \leq C\left(\|u\|_{B_{k, 2}^{\lambda}}+\|v\|_{B_{k, 2}^{\lambda}}\right)\left(\|u\|_{(p-1) s}^{p-1}+\|v\|_{(p-1) s}^{p-1}\right) .
\end{array}\right.
$$


Firstly, we consider $\|\cdot\|_{B_{k, 2}^{\lambda}}$. Since $\lambda=\sigma^{\prime \prime}+\delta(l) / n, 2 \leq l \leq \infty, \delta(r) \geq 1$, $\sigma^{\prime}=\varrho^{\prime}+\delta(r)-1$ and $0 \leq \sigma^{\prime}<\sigma^{\prime \prime} \leq \sigma^{\prime}+\varepsilon<1 / 2$, we have $\lambda \geq \sigma^{\prime \prime}>\sigma^{\prime} \geq \varrho^{\prime}$. We can use interpolation between $B_{r, 2}^{\varrho^{\prime}}$ and $H^{a}$ to estimate norms in $B_{k, 2}^{\lambda}$, where $H^{a} \sim\left(H^{1}, L^{2}\right)_{a}$ for $0<a \leq 1$, hence

$$
\lambda=\theta \varrho^{\prime}+(1-\theta) a \leq \theta \varrho^{\prime}+(1-\theta), \quad \frac{1}{k}=\frac{\theta}{r}+\frac{1-\theta}{2},
$$

i.e.,

$$
0 \leq \delta(k) \leq \frac{1-\lambda}{1-\varrho^{\prime}} \delta(r)
$$

Secondly, we consider $\|\cdot\|_{(p-1) s}$.

CAse 1: $(p-1) s \geq 2^{*}$.

SubCASE (i): $\varrho^{\prime}<0$. Note that

$$
L^{(p-1) s}=F_{(p-1) s, 2}^{0} \hookleftarrow B_{(p-1) s, 2}^{0} \sim\left(B_{r, 2}^{\varrho^{\prime}}, H^{1}\right)_{\theta}
$$

where

$$
0=\theta \varrho^{\prime}+(1-\theta), \quad \frac{1}{(p-1) s}=\frac{\theta}{r}+\frac{1-\theta}{2} .
$$

Hence

$$
\delta((p-1) s)=\frac{1}{1-\varrho^{\prime}} \delta(r)=1+\frac{\sigma^{\prime}}{1-\varrho^{\prime}} .
$$

SubCASE (ii): $\varrho^{\prime} \geq 0$. Noting that $\sigma^{\prime}=\varrho^{\prime}+\delta(r)-1,0 \leq \sigma^{\prime}<1 / 2$, we have

$$
-\frac{\varrho^{\prime}}{n}+\frac{1}{r}=\frac{1}{2}-\frac{1}{n}-\frac{\sigma^{\prime}}{n} \geq \frac{1}{2}-\frac{1}{n}-\frac{1}{2 n}=\frac{n-3}{2 n} \geq 0 .
$$

Define

$$
\frac{1}{\widetilde{r}}=\frac{1}{r}-\frac{\varrho^{\prime}}{n} .
$$

Then

$$
L^{(p-1) s} \sim\left(L^{\widetilde{r}}, L^{2}\right)_{\theta}
$$

where

$$
\frac{1}{(p-1) s}=\frac{\theta}{\widetilde{r}}+\frac{1-\theta}{2}, \quad 0 \leq \theta \leq 1 .
$$

Hence

$$
\delta((p-1) s)=\theta \delta(\widetilde{r})=\theta\left(\delta(r)+\varrho^{\prime}\right)=\theta\left(1+\sigma^{\prime}\right) \leq 1+\sigma^{\prime} .
$$

CASE 2: $(p-1) s<2^{*}$. Noting that $H^{1} \hookrightarrow L^{(p-1) s}$, we have

$$
\delta((p-1) s)<\delta\left(2^{*}\right)=1 \leq 1+\sigma^{\prime} .
$$


Combining the above two cases, we need the interpolation conditions

$$
\left\{\begin{array}{l}
0 \leq \delta(k) \leq \frac{1-\lambda}{1-\varrho^{\prime}} \delta(r), \\
0 \leq \delta((p-1) s) \leq 1+\min \left(\sigma^{\prime}, \frac{\sigma^{\prime}}{1-\varrho^{\prime}}\right)=1+\frac{\sigma^{\prime}}{1+\varrho_{-}^{\prime}},
\end{array}\right.
$$

where $\varrho_{ \pm}^{\prime}=\max \left(0, \pm \varrho^{\prime}\right)$, or equivalently

$$
\left\{\begin{array}{l}
0 \leq \delta(k) \leq \frac{1-\lambda}{1-\varrho^{\prime}} \delta(r), \\
(p-1)\left(\frac{n}{2}-1-\frac{\sigma^{\prime}}{1+\varrho_{-}^{\prime}}\right) \leq \frac{n}{s} \leq(p-1) \frac{n}{2},
\end{array}\right.
$$

which are satisfied provided

$$
\left\{\begin{array}{l}
(p-1) \frac{n}{2} \geq \delta(l) \\
(p-1)\left(\frac{n}{2}-1-\frac{\sigma^{\prime}}{1+\varrho_{-}^{\prime}}\right) \leq \delta(l)+\delta(r) \frac{1-\lambda}{1-\varrho^{\prime}} .
\end{array}\right.
$$

In fact, (3.33) and $n / s=\delta(l)+\delta(k)$ imply that

$$
(p-1)\left(\frac{n}{2}-1-\frac{\sigma}{1+\varrho_{-}^{\prime}}\right) \leq \delta(l)+\delta(k) \leq \frac{n}{2}(p-1),
$$

and we can always choose $k$ such that (3.33) holds under (3.34).

Since $\varrho \leq 1-\beta(r)$, a sufficient condition for $\varrho+\delta(r)-1<1 / 2$ is $1-\beta(r)+\delta(r)-1<1 / 2$, hence $\gamma(r)<1$ or $r<2(n-1) /(n-3)$. So

$$
\delta(l) \leq \delta(r) \leq \delta\left(\frac{2(n-1)}{n-3}\right)=\frac{n}{n-1}
$$

and the first interpolation condition is satisfied if $(p-1) \frac{n}{2} \geq \frac{n}{n-1}$, or equivalently

$$
p-1 \geq \frac{2}{n-1} .
$$

As to the second condition, note that $1+\sigma^{\prime}-\varrho^{\prime}=\delta(r), \sigma^{\prime \prime} \leq \sigma^{\prime}+\varepsilon$, $\lambda=\sigma^{\prime \prime}+\delta(l) / n$. A sufficient condition is

$$
(p-1)\left(\frac{n}{2}-1-\frac{\sigma^{\prime}}{1+\varrho_{-}^{\prime}}\right) \leq 1+\gamma(l)-\varepsilon-\frac{\sigma^{\prime}}{1-\varrho^{\prime}}\left(\delta(r)-1+\varepsilon+\frac{\delta(l)}{n}\right) .
$$

In fact, we only need to prove that

$$
1+\gamma(l)-\varepsilon-\frac{\sigma^{\prime}}{1-\varrho^{\prime}}\left(\delta(r)-1+\varepsilon+\frac{\delta(l)}{n}\right) \leq \delta(l)+\delta(r) \frac{1-\lambda}{1-\varrho^{\prime}}
$$

In view of the fact that 


$$
\begin{aligned}
1+\gamma(l)-\varepsilon-\frac{\sigma^{\prime}}{1-\varrho^{\prime}}(\delta(r)-1+ & \left.\varepsilon+\frac{\delta(l)}{n}\right) \\
& =\delta(r) \frac{1-\delta(l) / n-\varepsilon-\sigma^{\prime}}{1-\varrho^{\prime}}+\delta(l),
\end{aligned}
$$

and

$$
\delta(l)+\delta(r) \frac{1-\lambda}{1-\varrho^{\prime}}=\delta(l)+\delta(r) \frac{1-\sigma^{\prime \prime}-\delta(l) / n}{1-\varrho^{\prime}} .
$$

(3.37) is equivalent to

$$
\delta(r) \frac{1-\delta(l) / n-\varepsilon-\sigma^{\prime}}{1-\varrho^{\prime}}+\delta(l) \leq \delta(l)+\delta(r) \frac{1-\sigma^{\prime \prime}-\delta(l) / n}{1-\varrho^{\prime}},
$$

i.e., $\sigma^{\prime \prime} \leq \sigma^{\prime}+\varepsilon$, which is obviously true.

Now we begin to reduce condition (3.36). When $\sigma^{\prime}=0$, it reduces to

$$
p-1 \leq \frac{2(1+\gamma(l)-\varepsilon)}{n-2},
$$

which is satisfied for $\gamma(l)=1-\varepsilon$ provided $p-1 \leq 4(1-\varepsilon) /(n-2)$.

We only need to consider the case of $\sigma^{\prime}>0$. Under (3.41) we have

$$
(n-2)\left(\delta(r)-1+\varepsilon+\frac{\delta(l)}{n}\right) \leq 2(1+\gamma(l)-\varepsilon)\left(1-\varrho_{+}^{\prime}\right) .
$$

In fact, we only need to prove that

$$
\begin{aligned}
\frac{2(1+\gamma(l)-\varepsilon)}{n-2}\left(\frac{n}{2}\right. & \left.-1-\frac{\sigma^{\prime}}{1+\varrho_{-}^{\prime}}\right) \\
& \leq 1+\gamma(l)-\varepsilon-\frac{\sigma^{\prime}}{1-\varrho^{\prime}}\left(\delta(r)-1+\varepsilon+\frac{\delta(l)}{n}\right)
\end{aligned}
$$

while from

$$
\begin{aligned}
& \frac{2(1+\gamma(l)-\varepsilon)}{n-2}\left(\frac{n}{2}-1-\frac{\sigma^{\prime}}{1+\varrho_{-}^{\prime}}\right) \\
& \quad=1+\gamma(l)-\varepsilon-\frac{1+\gamma(l)-\varepsilon}{n-2} \frac{2 \sigma^{\prime}}{1+\varrho_{-}^{\prime}}
\end{aligned}
$$

we only need

$$
(1+\gamma(l)-\varepsilon) \frac{2 \sigma^{\prime}}{1+\varrho_{-}^{\prime}} \geq(n-2) \frac{\sigma^{\prime}}{1-\varrho^{\prime}}\left(\delta(r)-1+\varepsilon+\frac{\delta(l)}{n}\right),
$$

or equivalently

$$
2(1+\gamma(l)-\varepsilon)\left(1-\varrho_{+}^{\prime}\right) \geq(n-2)(\delta(r)-1+\varepsilon+\delta(l) / n)
$$

where we used $1-\varrho^{\prime}=\left(1-\varrho_{+}^{\prime}\right)\left(1+\varrho_{-}^{\prime}\right)$. 
Subcase $(\mathrm{i}): \varrho^{\prime} \leq 0$. Then $\varrho_{+}^{\prime}=0$ and (3.46) reduces to

$$
2(1+\gamma(l)-\varepsilon) \geq(n-2)(\delta(r)-1+\varepsilon+\delta(l) / n) .
$$

Since $\delta(r)=\frac{n}{n-1} \gamma(r)$, this is equivalent to

$$
(n-2)\left(\frac{n}{n-1} \gamma(r)-(1-\varepsilon)+\frac{\gamma(l)}{n-1}\right) \leq 2(1+\gamma(l)-\varepsilon) .
$$

By direct computation, we have

$$
(n-2) \gamma(r) \leq(n-1)(1-\varepsilon)+\gamma(l),
$$

which is satisfied by choosing $\gamma(l)=1-\varepsilon$ and $\gamma(r) \leq \frac{n}{n-2}(1-\varepsilon)$, and in particular for $\gamma(l)=\gamma(r)=1-\varepsilon$.

Subcase (ii): $\varrho^{\prime}>0$. Then $\varrho_{+}^{\prime}=\varrho^{\prime}$, and $\sigma^{\prime}=\varrho^{\prime}+\delta(r)-1$ implies $1-\varrho_{+}^{\prime}=1-\varrho^{\prime}=\delta(r)-\sigma^{\prime}$. Therefore (3.46) reduces to

$$
(n-2)(\delta(r)-1+\varepsilon+\delta(l) / n) \leq 2(1-\varepsilon+\gamma(l))\left(\delta(r)-\sigma^{\prime}\right) .
$$

Let $\gamma(r)=\gamma(l)=1-\varepsilon$. Using $\delta(r)=\frac{n}{n-1} \gamma(r)=\frac{n}{n-1}(1-\varepsilon)$, (3.50) reduces to

$$
(n-2) \leq 2 n(1-\varepsilon)-2 \sigma^{\prime}(n-1),
$$

which is satisfied for $0 \leq \sigma^{\prime}<1 / 2$, and for $\varepsilon \leq 3 / 2 n$.

Thus we have show that the interpolation is always possible. Since $1 / l^{\prime}=$ $1 / k+1 / s$, we can always choose $k$ and $s$ to ensure that $\lambda+\delta(k)-1$ and $\delta((p-1) s)-1$ have the same sign. In fact

$$
\left\{\begin{array}{l}
\lambda+\delta(k)-1=0 \text { implies } \frac{1}{k_{c}}=\frac{n-2(1-\lambda)}{2 n}, \\
\delta((p-1) s)-1=0 \text { implies } \frac{1}{s_{c}}=\frac{(n-2)(p-1)}{2 n} .
\end{array}\right.
$$

It is easy to see that

$$
\left\{\begin{array}{l}
1 / k>1 / k_{c} \Leftrightarrow \lambda+\delta(k)-1<0, \\
1 / k<1 / k_{c} \Leftrightarrow \lambda+\delta(k)-1>0, \\
1 / s>1 / s_{c} \Leftrightarrow \delta((p-1) s)-1<0, \\
1 / s<1 / s_{c} \Leftrightarrow \delta((p-1) s)-1>0 .
\end{array}\right.
$$

If $1 / k_{c}+1 / s_{c}<1 / l^{\prime}$, let $1 / l^{\prime}-\left(1 / k_{c}+1 / s_{c}\right)=\varepsilon>0$, and define

$$
\frac{1}{k}=\frac{1}{k_{c}}+\frac{\varepsilon}{2}>\frac{1}{k_{c}}, \quad \frac{1}{s}=\frac{1}{s_{c}}+\frac{\varepsilon}{2}>\frac{1}{s_{c}} .
$$

Then $\lambda+\delta(k)-1$ and $\delta((p-1) s)-1$ are both negative.

If $1 / k_{c}+1 / s_{c}>1 / l^{\prime}$, then we can also choose $k$ and $s$ to ensure that $\lambda+\delta(k)-1$ and $\delta((p-1) s)-1$ are both positive.

Now we begin to apply interpolation to prove the Claim; for this purpose, we consider the following two cases: 
Negative Case.

(i) $\delta((p-1) s)-1<0$, hence $(p-1) s<2^{*}$, therefore

$$
H^{1} \hookrightarrow L^{(p-1) s} .
$$

(ii) $\lambda+\delta(k)-1<0$, hence $B_{2,2}^{1} \hookrightarrow B_{k, 2}^{\lambda}$, therefore

$$
H^{1}=F_{2,2}^{1}=B_{2,2}^{1} \hookrightarrow B_{k, 2}^{\lambda} .
$$

Thus

$$
\|\varphi\|_{(p-1) s},\|\varphi\|_{B_{k, 2}^{\lambda}} \leq C\|\varphi\|_{H^{1}} .
$$

So

$$
\begin{aligned}
&\left\|f_{1}\left(|u|^{2}, v\right) u\right\|_{B_{l^{\prime}, 2}^{\lambda}},\left\|f_{2}\left(|u|^{2}, v\right)\right\|_{B_{l^{\prime}, 2}^{\lambda}} \\
& \leq C\left(\|u\|_{H^{1}}^{p-1}+\|v\|_{H^{1}}^{p-1}\right)\left(\|u\|_{H^{1}}+\|v\|_{H^{1}}\right) .
\end{aligned}
$$

\section{Positive Case.}

(i) $\delta((p-1) s)-1>0$, i.e., $(p-1) s>2^{*}$, hence

$$
L^{(p-1) s}=F_{(p-1) s, 2}^{0} \hookleftarrow B_{(p-1) s, 2}^{0} \sim\left(H^{1}, B_{r, 2}^{\varrho^{\prime}}\right)_{\theta_{1}} .
$$

(ii) $\lambda+\delta(k)-1>0$, i.e., $\delta(k)>1-\lambda$, hence

$$
B_{k, 2}^{\lambda} \sim\left(B_{2,2}^{1}, B_{r, 2}^{\varrho^{\prime}}\right)_{\theta_{2}}=\left(H^{1}, B_{r, 2}^{\varrho^{\prime}}\right)_{\theta_{2}} .
$$

So

$$
\begin{aligned}
\| f_{1}\left(|u|^{2}, v\right) & u \|_{B_{l^{\prime}, 2}^{\lambda}} \leq C\left(\|u\|_{(p-1) s}^{p-1}+\|v\|_{(p-1) s}^{p-1}\right)\left(\|u\|_{B_{k, 2}^{\lambda}}+\|v\|_{B_{k, 2}^{\lambda}}\right) \\
\leq & C\left(\|u\|_{H^{1}}^{(p-1) \theta_{1}}\|u\|_{B_{r, 2}^{e^{\prime}}}^{(p-1)\left(1-\theta_{1}\right)}+\|v\|_{H^{1}}^{(p-1) \theta_{1}}\|v\|_{B_{r, 2}^{e^{\prime}}}^{(p-1)\left(1-\theta_{1}\right)}\right) \\
& \cdot\left(\|u\|_{H^{1}}^{\theta_{2}}\|u\|_{B_{r, 2}^{e^{\prime}}}^{1-\theta_{2}}+\|v\|_{H^{1}}^{\theta_{2}}\|v\|_{B_{r, 2}^{e^{\prime}}}^{1-\theta_{2}}\right) \\
= & C\left(\|u\|_{H^{1}}^{(p-1) \theta_{1}+\theta_{2}}\|u\|_{B_{r, 2}^{\ell^{\prime}}}^{(p-1)\left(1-\theta_{1}\right)+\left(1-\theta_{2}\right)}\right. \\
& +\|v\|_{H^{1}}^{(p-1) \theta_{1}+\theta_{2}}\|v\|_{B_{r, 2}^{e^{\prime}}}^{(p-1)\left(1-\theta_{1}\right)+\left(1-\theta_{2}\right)} \\
& +\|u\|_{H^{1}}^{(p-1) \theta_{1}}\|u\|_{B_{r, 2}^{e^{\prime}}}^{(p-1)\left(1-\theta_{1}\right)}\|v\|_{H^{1}}^{\theta_{2}}\|v\|_{B_{r, 2}^{e^{\prime}}}^{1-\theta_{2}} \\
& \left.+\|v\|_{H^{1}}^{(p-1) \theta_{1}}\|v\|_{B_{r, 2}^{e^{\prime}}}^{(p-1)\left(1-\theta_{1}\right)}\|u\|_{H^{1}}^{e_{2}}\|u\|_{B_{r, 2}^{e^{\prime}}}^{1-\theta_{2}}\right) \\
\leq & C\left(\|u\|_{H^{1}}^{(p-1) \theta_{1}+\theta_{2}}+\|v\|_{H^{1}}^{(p-1) \theta_{1}+\theta_{2}}\right) \\
& \cdot\left(\|u\|_{B_{r, 2}^{e^{\prime}}}^{(p-1)\left(1-\theta_{1}\right)+\left(1-\theta_{2}\right)}+\|v\|_{B_{r, 2}^{e^{\prime}}}^{(p-1)\left(1-\theta_{1}\right)+\left(1-\theta_{2}\right)}\right) .
\end{aligned}
$$

Let $(p-1) \theta_{1}+\theta_{2}=p-\nu$. Then $(p-1)\left(1-\theta_{1}\right)+\left(1-\theta_{2}\right)=\nu$ and

$$
(p-1)(n / 2-1)=\delta(l)+1-\lambda+\nu \sigma^{\prime} .
$$


Since $\lambda=\sigma^{\prime \prime}+\delta(l) / n,(3.62)$ becomes

$$
\begin{aligned}
(p-1)(n / 2-1) & =\delta(l)+1-\sigma^{\prime \prime}-\delta(l) / n+\nu \sigma^{\prime} \\
& =\gamma(l)+1-\sigma^{\prime \prime}+\nu \sigma^{\prime} .
\end{aligned}
$$

The condition $\nu \leq 1$ becomes

$$
(p-1)(n / 2-1) \leq 1+\gamma(l)-\sigma^{\prime \prime}+\sigma^{\prime},
$$

which is satisfied by choosing $\gamma(l)=1-\varepsilon, \sigma^{\prime \prime} \leq \sigma^{\prime}+\varepsilon$ provided that $p-1 \leq 4(1-\varepsilon) /(n-2)$.

By (3.58) and (3.61),

$$
\begin{gathered}
\left\|U(t) f_{1}\left(|u|^{2}, v\right) u\right\|_{B_{r, 2}^{e^{\prime \prime}}} \leq C|t|^{-\delta(l)}\left\|f_{1}\left(|u|^{2}, v\right) u\right\|_{B_{l^{\prime}, 2}^{\lambda}} \\
\leq C|t|^{-\delta(l)}\left(\left(\|u\|_{H^{1}}^{p-\nu}+\|v\|_{H^{1}}^{p-\nu}\right)\left(\|u\|_{B_{r, 2}^{e^{\prime}}}^{\nu}+\|v\|_{B_{r, 2}^{e^{\prime}}}^{\nu}\right)\right. \\
\quad+\left(\|u\|_{H^{1}}^{p-1}+\|v\|_{H^{1}}^{p-1}\left(\|u\|_{H^{1}}+\|v\|_{H^{1}}\right)\right) \\
\leq C|t|^{-\delta(l)}\left(1+\|u\|_{B_{r, 2}^{e^{\prime}}}^{\nu}+\|v\|_{B_{r, 2}^{e^{\prime}}}^{\nu}\right) .
\end{gathered}
$$

By a similar argument, we have

$$
\left\|K(t) f_{2}\left(|u|^{2}, v\right)\right\|_{B_{r, 2}^{e^{\prime \prime}}} \leq C|t|^{-\gamma(l)}\left(1+\|u\|_{B_{r, 2}^{e^{\prime}}}^{\nu}+\|v\|_{B_{r, 2}^{e^{\prime}}}^{\nu}\right) .
$$

Now we use the Claim to complete the proof of Lemma 3.3 for $\gamma(r)=$ $1-\varepsilon$. We can easily obtain $\varrho_{m}=-(1-n \varepsilon) /(n-1)$, corresponding to $\sigma=0$, and $\varrho_{M}=\frac{1}{2}+\frac{(n+1) \varepsilon-2}{2(n-1)}$, corresponding to the upper limit allowed by the condition of Lemma 3.1. For that purpose, we estimate iteratively for $\varrho^{\prime \prime}$ increasing from $\varrho_{m}$ to $\varrho_{M}$ in steps of $\varepsilon$ and for $1 / q=\sigma^{\prime \prime}$, using the Claim. We obtain at each step

$$
\left\{\begin{aligned}
\|u(t)\|_{B_{r, 2}^{e^{\prime \prime}}} \leq & \|U(t) \varphi\|_{B_{r, 2}^{e^{\prime \prime}}}+C \int_{0}^{t}|t-\tau|^{-\delta(l)}\left(1+\|u\|_{B_{r, 2}^{e^{\prime}}}^{\nu}+\|v\|_{B_{r, 2}^{e^{\prime}}}^{\nu}\right) d \tau \\
\|v(t)\|_{B_{r, 2}^{e^{\prime \prime}}} \leq & \left\|\dot{K}(t) \psi_{1}+K(t) \psi_{2}\right\|_{B_{r, 2}^{e^{\prime \prime}}} \\
& +C \int_{0}^{t}|t-\tau|^{-\gamma(l)}\left(1+\|u\|_{B_{r, 2}^{e^{\prime}}}^{\nu}+\|v\|_{B_{r, 2}^{e^{\prime}}}^{\nu}\right) d \tau .
\end{aligned}\right.
$$

The norms of the constant terms were considered in Lemma 3.1. Applying the Young inequality to the time integral, we obtain, for any compact interval $J$ containing 0 , 


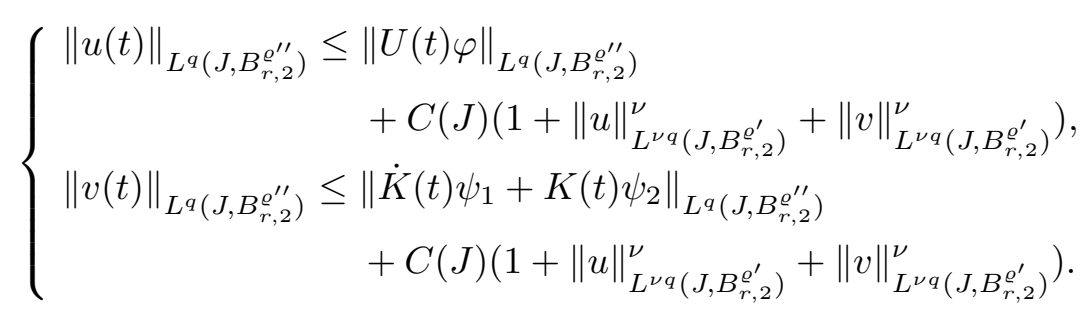

Since $\nu q \leq q<1 / \sigma^{\prime}$, we can continue this iteration; after a finite number of steps, we obtain $u(t), v(t) \in L^{q}\left(J, B_{r, 2}^{\varrho}\right)$ for all $\varrho \in\left[\varrho_{m}, \varrho_{M}\right]$ and $1 / q=\sigma$.

This completes the proof of the special case of $\gamma(r)=1-\varepsilon$. For $\gamma(\bar{r}) \geq 1$, let $\sigma=\bar{\varrho}+\delta(\bar{r})-1=\varrho+\delta(r)-1$ for $r \leq \bar{r}$. Then $B_{r, 2}^{\varrho} \hookrightarrow B_{\bar{r}, 2}^{\bar{\varrho}}$, therefore $\|\cdot\|_{B_{\overline{\bar{T}}, 2}^{\overline{\underline{a}}}} \leq C\|\cdot\|_{B_{r, 2}^{o}}$, so it can be reduced to the case of $\gamma(r)=1-\varepsilon$. Then

Proof of Theorem 2. Let $\left(u_{1}, v_{1}\right)$ and $\left(u_{2}, v_{2}\right)$ be two solutions of (1.1).

$$
\left\{\begin{array}{l}
u_{1}(t)-u_{2}(t)=-i \int_{0}^{t} U(t-\tau)\left(f_{1}\left(\left|u_{1}\right|^{2}, v_{1}\right) u_{1}-f_{1}\left(\left|u_{2}\right|^{2}, v_{2}\right) u_{2}\right) d \tau \\
v_{1}(t)-v_{2}(t)=-\frac{1}{2} \int_{0}^{t} K(t-\tau)\left(f_{2}\left(\left|u_{1}\right|^{2}, v_{1}\right)-f_{2}\left(\left|u_{2}\right|^{2}, v_{2}\right)\right) d \tau
\end{array}\right.
$$

We estimate (3.69) in $L^{\bar{r}}$ for some $\bar{r}$ satisfying

$$
0 \leq \bar{\gamma}=\gamma(\bar{r})=\frac{n-1}{n+1}, \quad 0 \leq \bar{\delta}=\delta(\bar{r})=\frac{n}{n+1} .
$$

On the one hand,

$$
\begin{aligned}
& \left\|U(t-\tau)\left(f_{1}\left(\left|u_{1}\right|^{2}, v_{1}\right) u_{1}-f_{1}\left(\left|u_{2}\right|^{2}, v_{2}\right) u_{2}\right)\right\|_{\bar{r}} \\
& \leq C|t-\tau|^{-\bar{\delta}}\left\|f_{1}\left(\left|u_{1}\right|^{2}, v_{1}\right) u_{1}-f_{1}\left(\left|u_{2}\right|^{2}, v_{2}\right) u_{2}\right\|_{\bar{r}^{\prime}} \\
& \leq C|t-\tau|^{-\bar{\delta}}\left(\left\|u_{1}\right\|_{(p-1) \bar{l}}^{p-1}+\left\|u_{2}\right\|_{(p-1) \bar{l}}^{p-1}+\left\|v_{1}\right\|_{(p-1) \bar{l}}^{p-1}+\left\|v_{2}\right\|_{(p-1) \bar{l}}^{p-1}\right) \\
& \quad \cdot\left(\left\|u_{1}-u_{2}\right\|_{\bar{r}}+\left\|v_{1}-v_{2}\right\|_{\bar{r}}\right)
\end{aligned}
$$

where $1 / \bar{r}^{\prime}=1 / \bar{r}+1 / \bar{l}$, so $2 \bar{\delta}=n / \bar{l}$. On the other hand,

$$
\begin{aligned}
\| K & (t-\tau)\left(f_{2}\left(\left|u_{1}\right|^{2}, v_{1}\right)-f_{2}\left(\left|u_{2}\right|^{2}, v_{2}\right)\right) \|_{\bar{r}} \\
\leq & C\left\|K(t-\tau)\left(f_{2}\left(\left|u_{1}\right|^{2}, v_{1}\right)-f_{2}\left(\left|u_{2}\right|^{2}, v_{2}\right)\right)\right\|_{B_{\bar{r}, 2}^{0}} \\
\leq & C|t-\tau|^{-\bar{\gamma}}\left\|f_{2}\left(\left|u_{1}\right|^{2}, v_{1}\right)-f_{2}\left(\left|u_{2}\right|^{2}, v_{2}\right)\right\|_{B_{\bar{r}^{\prime}, 2}^{2 \beta(\bar{r})-1}} \\
\leq & C|t-\tau|^{-\bar{\gamma}}\left\|f_{2}\left(\left|u_{1}\right|^{2}, v_{1}\right)-f_{2}\left(\left|u_{2}\right|^{2}, v_{2}\right)\right\|_{\bar{s}} \\
\leq & C|t-\tau|^{-\bar{\gamma}}\left(\left\|u_{1}\right\|_{(p-1) \bar{l}}^{p-1}+\left\|u_{2}\right\|_{(p-1) \bar{l}}^{p-1}+\left\|v_{1}\right\|_{(p-1) \bar{l}}^{p-1}+\left\|v_{2}\right\|_{(p-1) \bar{l}}^{p-1}\right) \\
& \cdot\left(\left\|u_{1}-u_{2}\right\|_{\bar{r}}+\left\|v_{1}-v_{2}\right\|_{\bar{r}}\right)
\end{aligned}
$$


where $B_{\bar{r}, 2}^{0} \hookrightarrow F_{\bar{r}, 2}^{0}=L^{\bar{r}}$ for $\bar{r} \geq 2$, and $L^{\bar{s}}=F_{\bar{s}, 2}^{0} \hookrightarrow B_{\bar{s}, 2}^{0} \hookrightarrow B_{\bar{r}^{\prime}, 2}^{2 \beta(\bar{r})-1}$. To ensure the embeddings, we need

$$
\bar{s} \leq \bar{r}^{\prime} \leq 2, \quad \frac{1}{\bar{s}}-\frac{1}{\bar{r}^{\prime}}=\frac{1-2 \beta(\bar{r})}{n},
$$

i.e.,

$$
\bar{s} \leq \bar{r}^{\prime} \leq 2, \quad \frac{n}{\bar{s}}=\frac{1}{\bar{r}}+\frac{n+1}{2},
$$

therefore $1 / \bar{r}+1 / \bar{l}=1 / \bar{s}$ implies

$$
\frac{n}{\bar{l}}=\frac{n}{\bar{s}}-\frac{n}{\bar{r}}=\frac{1}{\bar{r}}+\frac{n+1}{2}-\frac{n}{\bar{r}}=1+\bar{\gamma} .
$$

By the Hölder inequality, we obtain

$$
\left\{\begin{aligned}
\left\|u_{1}(t)-u_{2}(t)\right\|_{\bar{r}} \leq & C\left(\sup _{0 \leq \tau \leq t}\left\|u_{1}-u_{2}\right\|_{\bar{r}}+\sup _{0 \leq \tau \leq t}\left\|v_{1}-v_{2}\right\|_{\bar{r}}\right)|t|^{1-\bar{\delta}-1 / \bar{m}} \\
& \cdot\left(\left\|u_{1}\right\|_{(p-1) \bar{l},(p-1) \bar{m},[0, t]}^{p-1}+\left\|u_{2}\right\|_{(p-1) \bar{l},(p-1) \bar{m},[0, t]}^{p-1}\right. \\
& \left.+\left\|v_{1}\right\|_{(p-1) \bar{l},(p-1) \bar{m},[0, t]}^{p-1}+\left\|v_{2}\right\|_{(p-1) \bar{l},(p-1) \bar{m},[0, t]}^{p-1}\right), \\
\left\|v_{1}(t)-v_{2}(t)\right\|_{\bar{r}} \leq & C\left(\sup _{0 \leq \tau \leq t}\left\|u_{1}-u_{2}\right\|_{\bar{r}}+\sup _{0 \leq \tau \leq t}\left\|v_{1}-v_{2}\right\|_{\bar{r}}\right)|t|^{1-\bar{\gamma}-1 / \bar{m}} \\
& \cdot\left(\left\|u_{1}\right\|_{(p-1) \bar{l},(p-1) \bar{m},[0, t]}^{p-1}+\left\|u_{2}\right\|_{(p-1) \bar{l},(p-1) \bar{m},[0, t]}^{p-1}\right. \\
+ & \left.\left\|v_{1}\right\|_{(p-1) \bar{l},(p-1) \bar{m},[0, t]}^{p-1}+\left\|v_{2}\right\|_{(p-1) \bar{l},(p-1) \bar{m},[0, t]}^{p-1}\right) .
\end{aligned}\right.
$$

Hence we need $1 / \bar{m}<1-\bar{\delta}$ and $1 / \bar{m}<1-\bar{\gamma}$. Let $n / \bar{l}=2 \bar{\delta}=1+\bar{\gamma}$; then $\bar{\gamma}=(n-1) /(n+1), \bar{\delta}=n /(n+1)$.

If $(p-1) \bar{l} \leq 2^{*}$, we can simply use $H^{1} \hookrightarrow L^{(p-1) \bar{l}}$. It remains to consider the case of $(p-1) \bar{l}>2^{*}$. This time we turn to Lemma 3.3 for $\varrho=0$, therefore we need some $r$ such that

$$
\left\{\begin{array}{l}
1 \leq \delta(r)<3 / 2, \quad 2 \leq(p-1) \bar{l} \leq r \\
\delta((p-1) \bar{l})-1 \leq \frac{1}{(p-1) \bar{m}}
\end{array}\right.
$$

or equivalently

$$
\left\{\begin{array}{l}
1 \leq \delta(r)<3 / 2 \\
\frac{2}{\bar{l}} \leq p-1 \leq \frac{r}{\bar{l}}=\frac{1+\bar{\gamma}}{n} r=\frac{2 \bar{\delta}}{n} r \\
\frac{1}{\bar{m}} \geq \frac{n}{2}(p-1)-\frac{n}{\bar{l}}-(p-1)=\left(\frac{n}{2}-1\right)(p-1)-\frac{n}{\bar{l}} .
\end{array}\right.
$$

Firstly, to prove

$$
(p-1)\left(\frac{n}{2}-1\right)-\frac{n}{\bar{l}} \leq \frac{1}{\bar{m}}<1-\bar{\delta}<1-\bar{\gamma},
$$


it is sufficient to consider

$$
(p-1)(n / 2-1)-2 \bar{\delta}<1-\bar{\delta}
$$

i.e.,

$$
p-1<\frac{2(1+\bar{\delta})}{n-2}=\frac{4}{n-2}-\frac{2}{(n+1)(n-2)} .
$$

Secondly, we still need to prove that

$$
p-1 \leq \frac{1+\bar{\gamma}}{n} r
$$

since $\frac{r}{n}=\frac{2}{n-2 \delta(r)}$, it becomes

$$
p-1 \leq \frac{2(1+\bar{\gamma})}{n-2 \delta(r)},
$$

which follows from (H3) provided $(1+\bar{\gamma})(n-2) \geq 2(n-2 \delta(r))$, a condition which is satisfied by choosing $\bar{\gamma}=(n-1) /(n+1)$ and $\delta(r)=3 n / 2(n+1)<$ $3 / 2$.

Taking in (3.75) the supremum over $t$ in a sufficiently small interval containing 0 , we obtain a linear inequality which implies that $\left(u_{1}, v_{1}\right)=\left(u_{2}, v_{2}\right)$ in that interval. Iterating the process yields $\left(u_{1}, v_{1}\right)=\left(u_{2}, v_{2}\right)$ everywhere in $I$.

THEOREM 3.2. The solution of (1.1) satisfies the conservation of energy

$$
E(u, v, \dot{v})=E\left(\varphi, \psi_{1}, \psi_{2}\right)
$$

Proof. By the uniqueness of the solution $(u, v)$, we can apply time reversal to system (1.1) and therefore to the energy inequality, thereby completing the proof of energy conservation (3.83).

4. Improvement of the uniqueness. Though the method presented in $\S 3$ is very simple in form, the restriction on $p$ is unnatural. By choosing two sets of special admissible pairs $(r, q)$, we will use a special Strichartz estimate to remove the unnatural restriction on $p$ under the more general assumption $\left(\mathrm{H}^{\prime}\right)$ instead of (H3) in this section. Clearly (H3') implies $\left(\mathrm{H}^{\prime}{ }^{\prime}\right)$ and $p<1+4 /(n-2)$ implies that $H^{1} \hookrightarrow L^{p+1}$, so $X=H^{1}$.

Proof of Theorem 3. To reduce the length of the paper, we will be brief. Let $\left(u_{1}, v_{1}\right)$ and $\left(u_{2}, v_{2}\right)$ be two solutions of $(1.1)$. Then

$$
\begin{aligned}
& u_{1}(t)-u_{2}(t)=-i \int_{0}^{t} U(t-\tau)\left(f_{1}\left(\left|u_{1}\right|^{2}, v_{1}\right) u_{1}-f_{1}\left(\left|u_{2}\right|^{2}, v_{2}\right) u_{2}\right) d \tau \\
& v_{1}(t)-v_{2}(t)=-\frac{1}{2} \int_{0}^{t} K(t-\tau)\left(f_{2}\left(\left|u_{1}\right|^{2}, v_{1}\right)-f_{2}\left(\left|u_{2}\right|^{2}, v_{2}\right)\right) d \tau
\end{aligned}
$$


Taking $r_{1}=p+1$, we have

$$
\frac{2}{q_{1}}=\delta\left(r_{1}\right), \frac{1}{r_{2}}=\frac{n+1}{2(n-1)}-\frac{n-2}{2(n-1)}(p-1), \frac{2}{q_{2}}=\gamma\left(r_{2}\right)=(n-1)\left(\frac{1}{2}-\frac{1}{r_{2}}\right) .
$$

Thus $1-2 / q_{1}>0$ and $1-2 / q_{2}>0$.

Let $X=L^{q_{1}}\left(I, L^{r_{1}}\right) \cap L^{\infty}\left(I, L^{2}\right), Y=L^{q_{2}}\left(I, L^{r_{2}}\right) \cap L^{\infty}\left(I, L^{2}\right)$ and $Z=$ $X \times Y$. We estimate (4.1) and (4.2) in $X$ and $Y$ respectively. Firstly,

$$
\begin{aligned}
= & \left\|-i \int U(t-\tau)\left(f_{1}\left(\left|u_{1}\right|^{2}, v_{1}\right) u_{1}-f_{1}\left(\left|u_{2}\right|^{2}, v_{2}\right) u_{2}\right) d \tau\right\|_{L^{q_{1}\left(I, L^{r_{1}}\right) \cap L^{\infty}\left(I, L^{2}\right)}} \\
\leq & C\left\|\left(\left|u_{1}\right|^{p-1}+\left|u_{2}\right|^{p-1}+\left|v_{1}\right|^{p-1}+\left|v_{2}\right|^{p-1}\right)\left(\left|u_{1}-u_{2}\right|+\left|v_{1}-v_{2}\right|\right)\right\|_{L^{q_{1}^{\prime}\left(I, L^{\prime}\right.} r_{1}^{\prime}} \\
& +\left\|u_{1}-u_{2}\right\|_{L^{1}\left(I, L^{2}\right)}+\left\|v_{1}-v_{2}\right\|_{L^{1}\left(I, L^{2}\right)} \\
\leq & C\left\|\left(\left|u_{1}\right|^{p-1}+\left|u_{2}\right|^{p-1}+\left|v_{1}\right|^{p-1}+\left|v_{2}\right|^{p-1}\right)\left|u_{1}-u_{2}\right|\right\|_{L^{q_{1}^{\prime}\left(I, L^{r_{1}^{\prime}}\right)}} \\
& +C\left\|\left(\left|u_{1}\right|^{p-1}+\left|u_{2}\right|^{p-1}+\left|v_{1}\right|^{p-1}+\left|v_{2}\right|^{p-1}\right)\left|v_{1}-v_{2}\right|\right\|_{L^{q_{1}^{\prime}\left(I, L^{r_{1}^{\prime}}\right)}} \\
& +C|I|\left(\left\|u_{1}-u_{2}\right\|_{L^{\infty}\left(I, L^{2}\right)}+\left\|v_{1}-v_{2}\right\|_{L^{\infty}\left(I, L^{2}\right)}\right) \\
= & I_{1}+I_{2}+I_{3} .
\end{aligned}
$$

Now,

$$
\begin{aligned}
& I_{1}=C\left\|\left(\left|u_{1}\right|^{p-1}+\left|u_{2}\right|^{p-1}+\left|v_{1}\right|^{p-1}+\left|v_{2}\right|^{p-1}\right)\left|u_{1}-u_{2}\right|\right\|_{L^{q_{1}^{\prime}\left(I, L^{r_{1}^{\prime}}\right)}} \\
\leq & C\left\|\left(\left\|u_{1}\right\|_{L^{p+1}}^{p-1}+\left\|u_{2}\right\|_{L^{p+1}}^{p-1}+\left\|v_{1}\right\|_{L^{p+1}}^{p-1}+\left\|v_{2}\right\|_{L^{p+1}}^{p-1}\right)\right\| u_{1}-u_{2}\left\|_{L^{p+1}}\right\|_{L^{q_{1}^{\prime}}} \\
\leq & C\left(\left\|u_{1}\right\|_{L^{\infty}\left(I, H^{1}\right)}^{p-1}+\left\|u_{2}\right\|_{L^{\infty}\left(I, H^{1}\right)}^{p-1}+\left\|v_{1}\right\|_{L^{\infty}\left(I, H^{1}\right)}^{p-1}+\left\|v_{2}\right\|_{L^{\infty}\left(I, H^{1}\right)}^{p-1}\right) \\
& \cdot|I|^{1-2 / q_{1}}\left\|u_{1}-u_{2}\right\|_{L^{q_{1}\left(I, L^{r_{1}}\right)}}
\end{aligned}
$$

where we used $H^{1} \hookrightarrow L^{p+1}$ and the Hölder inequalities with

$$
\frac{1}{r_{1}^{\prime}}=\frac{1}{(p+1)^{\prime}}=1-\frac{1}{p+1}=\frac{p}{p+1}=\frac{p-1}{p+1}+\frac{1}{p+1},
$$

and

$$
\frac{1}{q_{1}^{\prime}}=\frac{1}{q_{1}}+\frac{1}{\chi}, \quad \text { so } \quad \frac{1}{\chi}=1-\frac{2}{q_{1}}
$$

As to $I_{2}$, since

$$
\begin{aligned}
1-\frac{1}{p+1} & =\frac{1}{(p+1)^{\prime}} \geq \frac{p-1}{2^{*}}+\frac{1}{r_{2}} \\
& =(p-1) \frac{n-2}{2 n}+\left[\frac{n+1}{2(n-1)}-\frac{n-2}{2(n-1)}(p-1)\right] \\
& =-\frac{n-2}{2 n(n-1)}(p-1)+\frac{n+1}{2(n-1)}
\end{aligned}
$$


it is easy to deduce from $p-1<\frac{4}{n-2}$ that for sufficiently small $\varepsilon$,

$$
p-1 \leq \frac{4-2 \varepsilon}{n-2}, \quad p+1 \leq \frac{2 n-2 \varepsilon}{n-2},
$$

and then (4.7) becomes

$$
\begin{aligned}
\frac{n+2-2 \varepsilon}{2 n-2 \varepsilon} & =1-\frac{n-2}{2 n-2 \varepsilon} \geq 1-\frac{1}{p+1} \\
& \geq-\frac{n-2}{2 n(n-1)}(p-1)+\frac{n+1}{2(n-1)} \\
& \geq-\frac{n-2}{2 n(n-1)} \frac{4-2 \varepsilon}{n-2}+\frac{n+1}{2(n-1)} \\
& =-\frac{4-2 \varepsilon}{2 n(n-1)}+\frac{n+1}{2(n-1)}=\frac{n^{2}+n-4+2 \varepsilon}{2 n(n-1)} .
\end{aligned}
$$

If we let $\varepsilon \rightarrow 0,(4.9)$ reduces to $n^{2}+n-2 \geq n^{2}+n-4$, which is obviously true.

Thus

$$
\begin{aligned}
\text { (4.10) } I_{2} \leq & C\left\|\left(\left\|u_{1}\right\|_{H^{1}}^{p-1}+\left\|u_{2}\right\|_{H^{1}}^{p-1}+\left\|v_{1}\right\|_{H^{1}}^{p-1}+\left\|v_{2}\right\|_{H^{1}}^{p-1}\right)\right\| v_{1}-v_{2}\left\|_{r_{2}}\right\|_{L^{q_{1}^{\prime}}} \\
\leq & C\left(\left\|u_{1}\right\|_{L^{\infty}\left(I, H^{1}\right)}^{p-1}+\left\|u_{2}\right\|_{L^{\infty}\left(I, H^{1}\right)}^{p-1}+\left\|v_{1}\right\|_{L^{\infty}\left(I, H^{1}\right)}^{p-1}+\left\|v_{2}\right\|_{L^{\infty}\left(I, H^{1}\right)}^{p-1}\right) \\
& \cdot|I|^{1-1 / q_{1}-1 / q_{2}}\left\|v_{1}-v_{2}\right\|_{L^{q_{2}\left(I, L^{r_{2}}\right)}}
\end{aligned}
$$

where

$$
\frac{1}{q_{1}^{\prime}}=\frac{1}{q_{2}}+\frac{1}{\chi}, \quad \text { so } \quad \frac{1}{\chi}=\frac{1}{q_{1}^{\prime}}-\frac{1}{q_{2}}=1-\frac{1}{q_{1}}-\frac{1}{q_{2}} .
$$

Secondly, by the Strichartz estimate [16],

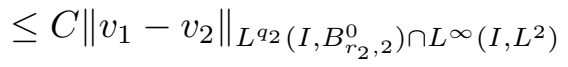

$$
\begin{aligned}
& =C\left\|-\frac{1}{2} \int K(t-\tau)\left(f_{2}\left(\left|u_{1}\right|^{2}, v_{1}\right)-f_{2}\left(\left|u_{2}\right|^{2}, v_{2}\right)\right) d \tau\right\|_{L^{q_{2}\left(I, B_{r_{2}, 2}^{0}\right) \cap L^{\infty}\left(I, L^{2}\right)}} \\
& \leq C\left\|\left(\left|u_{1}\right|^{p-1}+\left|u_{2}\right|^{p-1}+\left|v_{1}\right|^{p-1}+\left|v_{2}\right|^{p-1}\right)\left|u_{1}-u_{2}\right|\right\|_{L^{1}\left(I, B_{2,2}^{-1}\right)} \\
& +C\left\|\left(\left|u_{1}\right|^{p-1}+\left|u_{2}\right|^{p-1}+\left|v_{1}\right|^{p-1}+\left|v_{2}\right|^{p-1}\right)\left|v_{1}-v_{2}\right|\right\|_{L_{2}^{q_{2}^{\prime}\left(I, B_{r_{2}, 2}^{2 \beta\left(r_{2}\right)-1}\right)}} \\
& +C|I|\left(\left\|u_{1}-u_{2}\right\|_{L^{\infty}\left(I, L^{2}\right)}+\left\|v_{1}-v_{2}\right\|_{L^{\infty}\left(I, L^{2}\right)}\right) \\
& =I_{1}+I_{2}+I_{3} \text {. }
\end{aligned}
$$

Now, 
$(4.13)$

$$
\begin{aligned}
I_{1}= & C\left\|\left(\left|u_{1}\right|^{p-1}+\left|u_{2}\right|^{p-1}+\left|v_{1}\right|^{p-1}+\left|v_{2}\right|^{p-1}\right)\left|u_{1}-u_{2}\right|\right\|_{L^{1}\left(I, B_{2,2}^{-1}\right)} \\
\leq & C\left\|\left(\left|u_{1}\right|^{p-1}+\left|u_{2}\right|^{p-1}+\left|v_{1}\right|^{p-1}+\left|v_{2}\right|^{p-1}\right)\left|u_{1}-u_{2}\right|\right\|_{L^{1}\left(I, L^{\left(2^{*}\right)^{\prime}}\right)} \\
\leq & C\left(\left\|u_{1}\right\|_{L^{\infty}\left(I, H^{1}\right)}^{p-1}+\left\|u_{2}\right\|_{L^{\infty}\left(I, H^{1}\right)}^{p-1}+\left\|v_{1}\right\|_{L^{\infty}\left(I, H^{1}\right)}^{p-1}+\left\|v_{2}\right\|_{L^{\infty}\left(I, H^{1}\right)}^{p-1}\right) \\
& \cdot|I|^{1-1 / q_{1}}\left\|u_{1}-u_{2}\right\|_{L^{q_{1}\left(I, L^{r_{1}}\right)}}
\end{aligned}
$$

where

$$
\frac{1}{1}=\frac{1}{q_{1}}+\frac{1}{\chi}, \quad \text { so } \quad \frac{1}{\chi}=1-\frac{1}{q_{1}}
$$

and

$$
\frac{1}{\left(2^{*}\right)^{\prime}}=\frac{n+2}{2 n} \geq(p-1) \frac{n-2}{2 n}+\frac{1}{p+1}=\frac{p-1}{2^{*}}+\frac{1}{r_{1}}
$$

i.e.,

$$
1 \geq p \frac{n-2}{2 n}+\frac{1}{p+1},
$$

which is obviously true for $1 \leq p<(n+2) /(n-2)$. Moreover,

$$
\begin{aligned}
& I_{2}=C \|\left(\left|u_{1}\right|^{p-1}+\left|u_{2}\right|^{p-1}+\left|v_{1}\right|^{p-1}+\left|v_{2}\right|^{p-1}\right) \\
& \cdot\left|v_{1}-v_{2}\right| \|_{L^{q_{2}^{\prime}\left(I, B_{r_{2}^{\prime}, 2}^{2 \beta\left(r_{2}\right)-1}\right)}} \\
& \leq C\left(\left\|u_{1}\right\|_{L^{\infty}\left(I, H^{1}\right)}^{p-1}+\left\|u_{2}\right\|_{L^{\infty}\left(I, H^{1}\right)}^{p-1}+\left\|v_{1}\right\|_{L^{\infty}\left(I, H^{1}\right)}^{p-1}+\left\|v_{2}\right\|_{L^{\infty}\left(I, H^{1}\right)}^{p-1}\right) \\
& \quad \cdot|I|^{1-2 / q_{2}}\left\|v_{1}-v_{2}\right\|_{L^{q_{2}\left(I, L^{r_{2}}\right)}}
\end{aligned}
$$

where

$$
\frac{1}{q_{2}^{\prime}}=\frac{1}{q_{2}}+\frac{1}{\chi}, \quad \text { so } \quad \frac{1}{\chi}=\frac{1}{q_{2}^{\prime}}-\frac{1}{q_{2}}=1-\frac{2}{q_{2}}
$$

and

$$
\frac{1}{r_{2}^{\prime}}-\frac{2 \beta\left(r_{2}\right)-1}{n}=\frac{p-1}{2^{*}}+\frac{1}{r_{2}},
$$

which determines the choice of the $r_{2}$.

So

$$
\begin{gathered}
\left\|\left(u_{1}(t), v_{1}(t)\right)-\left(u_{2}(t), v_{2}(t)\right)\right\|_{Z}=\left\|u_{1}(t)-u_{2}(t)\right\|_{X}+\left\|v_{1}(t)-v_{2}(t)\right\|_{Y} \\
\leq C|I|^{\min \left(1-2 / q_{1}, 1-2 / q_{2}\right)}\left\|\left(u_{1}(t), v_{1}(t)\right)-\left(u_{2}(t), v_{2}(t)\right)\right\|_{Z} \\
=a\left\|\left(u_{1}(t), v_{1}(t)\right)-\left(u_{2}(t), v_{2}(t)\right)\right\|_{Z}
\end{gathered}
$$

Choosing $I$ small enough to ensure that $a<1$, we obtain the uniqueness of (1.1) in this tiny interval; iterating this process we get the uniqueness for all $t \in \mathbb{R}$. 
Acknowledgements. The authors are grateful to Professor Xin Zhouping for his careful comments and valuable suggestions.

\section{References}

[1] A. Bachelot, Problème de Cauchy pour des systèmes hyperboliques semi-linéaires, Ann. Inst. H. Poincaré Anal. Non Linéaire 1 (1984), 453-478.

[2] J.-B. Baillon and J. M. Chadam, The Cauchy problem for the coupled SchrödingerKlein-Gordon equations, in: Contemporary Developments in Continuum Mechanics and PDE, North-Holland, Amsterdam, 1978, 37-44.

[3] J. Bourgain, Refinements of Strichartz' inequality and applications to 2D-NLS with critical non-linearity, Int. Math. Res. Not. 1998, no. 5, 253-283.

[4] - Scattering in the energy space and below for 3D-NLS, J. Anal. Math. 75 (1998), 267-297.

[5] P. Brenner and W. Wahl, Global classical solutions of nonlinear wave equations, Math. Z. 176 (1981), 87-121.

[6] H. Brezis and T. Gallouët, Nonlinear Schrödinger evolution equations, J. Nonlinear Anal. 4 (1980), 677-681.

[7] H. Brezis and S. Wainger, A note on limiting cases of Sobolev embedding, Comm. Partial Differential Equations 5 (1980), 773-789.

[8] I. Fukuda and M. Tsutsumi, On coupled Klein-Gordon-Schrödinger equations III, Math. Japon. 24 (1979), 307-321.

[9] - - - On coupled Klein-Gordon-Schrödinger equations II, J. Math. Anal. Appl. 66 (1978), 358-378.

[10] J. Ginibre and G. Velo, The global Cauchy problem for the nonlinear Klein-Gordon equation, Math. Z. 189 (1985), 487-505.

[11] —, - , The global Cauchy problem for the nonlinear Schrödinger equation revisited, Ann. Inst. H. Poincaré Anal. Non Linéaire 2 (1985), 309-327.

[12] B. L. Guo and C. X. Miao, The global existence and asymptotic behavior of solutions for the coupled Klein-Gordon-Schrödinger equations, Sci. China Ser. A 38 (1995), $1444-1456$.

[13] P. Hartman, Ordinary Differential Equations, Birkhäuser, Boston, 1982.

[14] N. Hayashi and W. Wahl, On the global strong solutions of coupled Klein-GordonSchrödinger equations, J. Math. Soc. Japan 39 (1987), 489-497.

[15] J.-L. Lions, Quelques méthodes de résolution des problèmes aux limites non-linéaires, Dunod, Paris, 1969.

[16] C. X. Miao, Harmonic Analysis and Applications to PDE, 2nd ed., Science Press, 2004.

[17] T. Ozawa and Y. Tsutsumi, Asymptotic behaviour of solutions for the coupled KleinGordon-Schrödinger equations, Adv. Stud. Pure Math. 23 (1994), 295-305.

[18] H. Pecher, Global solutions of the Klein-Gordon-Schrödinger system with rough data, Differential Integral Equations 17 (2004), 179-214.

[19] W. Wahl, Analytische Abbildungen und semilineare Differentialgleichungen in Banachräumen, Nachr. Akad. Wiss. Göttingen II: Math. Phys Kl. 1979, 153-200.

[20] - Nichtlineare Evolutionsgleichungen, in: Teubner Texte Math. 50, Leipzig, 1983, 294-302. 
[21] W. Wahl, Über das Verhalten für $t \rightarrow 0$ der Lösungen nichtlinearer parabolischer Gleichungen, insbesondere der Gleichungen von Navier-Stokes, Bayreuth. Math. Schr. 16 (1984), 151-277.

[22] B. X. Wang, Classical global solutions for non-linear Klein-Gordon-Schrödinger equations, Math. Methods Appl. Sci. 20 (1997), 599-616.

Institute of Applied Physics and Computational Mathematics

P.O. Box 8009, Beijing 100088, China

E-mail: miao_changxing@iapcm.ac.cn

youbinzhu@yahoo.com.cn

Received 27.2.2006 